The University of Southern Mississippi

The Aquila Digital Community

Master's Theses

Spring 5-2014

\title{
Minority Health Perceptions in the Lower Mississippi Delta: A Grounded Theory Study Using PhotoVoice Methodology
}

SaVanna Beth Sims

University of Southern Mississippi

Follow this and additional works at: https://aquila.usm.edu/masters_theses

\section{Recommended Citation}

Sims, SaVanna Beth, "Minority Health Perceptions in the Lower Mississippi Delta: A Grounded Theory Study Using PhotoVoice Methodology" (2014). Master's Theses. 23.

https://aquila.usm.edu/masters_theses/23

This Masters Thesis is brought to you for free and open access by The Aquila Digital Community. It has been accepted for inclusion in Master's Theses by an authorized administrator of The Aquila Digital Community. For more information, please contact Joshua.Cromwell@usm.edu. 
The University of Southern Mississippi

MINORITY HEALTH PERCEPTIONS IN THE LOWER MISSISSIPPI

DELTA: A GROUNDED THEORY STUDY USING

PHOTOVOICE METHODOLOGY

by

SaVanna Beth Sims

A Thesis

Submitted to the Graduate School

of The University of Southern Mississippi

in Partial Fulfillment of the Requirements

for the Degree of Master of Science

Approved:

Holly F. Huye

Director

_Carol L. Connell

_Alicia S. Landry

Maureen A. Ryan

Dean of the Graduate School

May 2014 


\section{ABSTRACT \\ MINORITY HEALTH PERCEPTIONS IN THE LOWER MISSISSIPPI \\ DELTA: A GROUNDED THEORY STUDY USING \\ PHOTOVOICE METHODOLOGY \\ by SaVanna Beth Sims}

May 2014

The Lower Mississippi Delta (LMD) region has a large minority population with concentrations of poverty and health disparities much higher than other parts of the country. The purpose of this project was to assess the health perceptions of minority women living in the LMD using a combination of PhotoVoice and journal reflections. After an initial interview, 10 women were provided digital cameras and asked to take photographs that reflected the health of their communities. A final interview was conducted to glean more information from the women about their photographs. Seven Black women and two Latinas completed the study. The photographs, journal reflections, and transcripts collected during the study were analyzed using grounded theory principles. The core category identified by the researchers was Hope for a Healthy Future. The participants were optimistic about the future of the LMD and were striving to attain the Ideal Healthy Community, which consisted of five pillars: Access to Healthy Food, Nutrition Education, Physical Activity, Safety and Cleanliness, and Role Models. Participants identified barriers and facilitators to each of these pillars in their communities. The primary facilitator to health was Spiritual, Familial, and Community Ties. The primary barrier to health was Resistance to Change. Overall, striving towards the Ideal Healthy Community was a source of participants' Hope for a Healthy Future. 
Findings from this study can be used to further understand the health-related needs of minorities in the LMD and to advocate for positive change in LMD communities. 


\section{ACKNOWLEDGMENTS}

There are so many people who have made this project possible. First, I would like to thank Dr. Holly Huye, my research advisor, for her guidance and support over the past several months. This project was her brainchild, and I have been honored to be a part of it. I would also like to thank Dr. Carol Connell and Dr. Alicia Landry for serving on my thesis committee and for providing their insights. They are both so incredibly intelligent! I would also like to thank LaShaundrea Crook for her input, as well as Rebecca Bishop. Rebecca, I am so glad that there was someone in the office to share this experience with me! I would also like to extend my gratitude to the Committee on Services and Resources for Women, who provided funding for this project in the form of a grant.

My fiancé, Nathaniel Shoemaker, has been an invaluable source of inspiration and support throughout this process, as have my parents, John and Kimberly Sims, and my sister, Allie Sims. I love you all very much, and you have no idea how much your love and encouragement has meant to me.

Finally, I want to extend my deepest thanks to the women who participated in this project. You ladies are remarkable role models for the residents of the Lower Mississippi Delta, and I hope that this project helps you all to continue to improve the health of your communities. 


\section{TABLE OF CONTENTS}

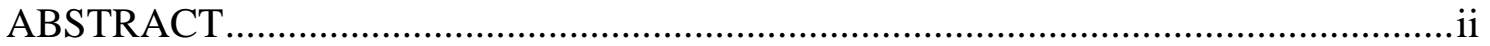

ACKNOWLEDGMENTS ................................................................................ iv

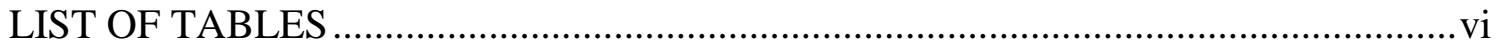

LIST OF ILLUSTRATIONS ...................................................................... vii

\section{CHAPTER}

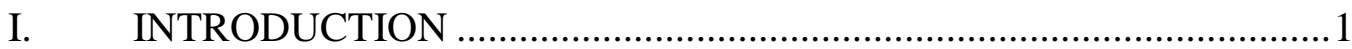

Background and Significance

Research Questions

Theoretical Framework

II. REVIEW OF RELATED LITERATURE ........................................4

The Lower Mississippi Delta

Developing Culturally Appropriate Interventions

PhotoVoice

Grounded Theory

Conclusion

III. METHODOLOGY

Research Design

Participants

Data Collection

Data Analysis

Framework Development

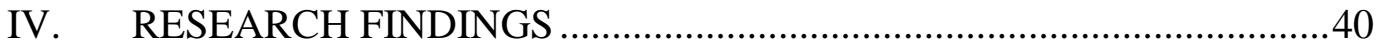

Demographic Information

Initial Interview

Conceptual Framework

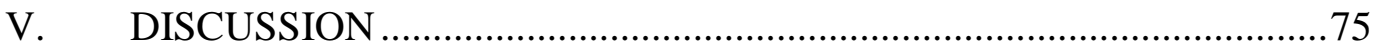

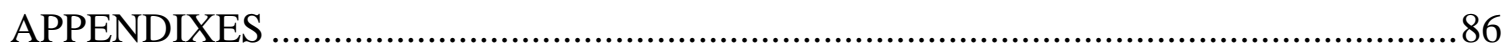

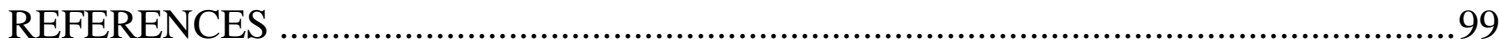




\section{LIST OF TABLES}

Table

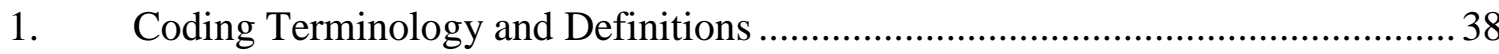

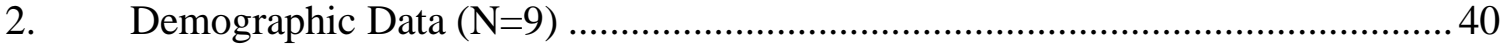

3. Participants' Health Concerns from Initial Interview ..................................... 41 


\section{LIST OF ILLUSTRATIONS}

Figure

1. A model for merged PhotoVoice and grounded theory projects ...................... 32

2. Conceptual framework: Health perceptions of minority women living in the LMD

3. Participants were concerned about creating a healthy future for children ........... 46

4. Participants were particularly interested in home gardening, community gardening, and farmer's markets....

5. Some of the participants were involved in community gardening programs through schools 48

6. Locally grown foods were important to participants because they were healthy and they kept money in the LMD's economy....

7. Participants perceived that many people in the LMD ate unhealthy foods because they were more convenient than healthy foods

8. Participants noticed a definite difference in the food preferences of the older generation and the younger generation

9. Participants shared how easy it was to get fried food in the LMD and also that many people probably did not realize that fried foods were unhealthy

10. Many participants were involved in nutrition education activities through schools, churches, or community organizations .53

11. There were many recreational facilities, like playgrounds and parks, in the LMD

12. Participants took many photographs of good examples of safety and cleanliness in their communities.

13. Participants took many photographs of abandoned homes, buildings, and cars. They perceived these abandoned facilities as dangerous to all community members, but especially to children.

14. Unkempt grass, broken equipment, and litter were some of the most photographed subjects related to safety and cleanliness 
15. Participants wanted to lead healthy lives so that they could be role models to future generations, like this woman.

16. These photographs, of a man who lost 60 pounds and his niece, highlight the importance of healthy role models in the LMD

17. Prayer and spirituality were very important to the Black participants. .62

18. Many photographs illustrated the importance of family

19. Family members were a source of encouragement, and they also enforced healthy behaviors when necessary

20. Churches were the cornerstones of LMD communities, according to participants .65

21. School functions allowed opportunities for both children and adults to engage in physical activity 66

22. Participants took many photographs of health promotion organizations and activities .67

23. In some instances, adopting American foods helped the Latina participants to be healthier

24. Mexican restaurants often prepared their foods "American-style," making them very unhealthy

25. Friendships were important to the Latina participants, and these friends encouraged each other to engage in healthy behaviors

26. A Latina participant instilled healthy habits in her children by having them exercise after eating a big meal

27. Cooking and eating traditional foods was perceived by the Latina participants as much healthier than American foods

28. The Latina participants tried to maintain their cultural food traditions by cooking Mexican and Ecuadorian foods at home

29. A typical Latino breakfast of coffee and crackers is much smaller than a standard American breakfast containing eggs and toast. 


\section{CHAPTER I}

\section{INTRODUCTION}

A common passion for the "health of the Delta" has become evident through focus groups with women living in the Lower Mississippi Delta (LMD) region. (Huye, Connell, Crook, Yadrick, \& Zoellner, 2014). As a result, the "Health of the Delta" PhotoVoice project was developed as a way to assess the health perceptions and healthrelated desires of women living in the LMD using visual depiction and written reflection. The project was expanded to include the Latina voice alongside the Black voice in order to gain the perspective of the two largest ethnic minority groups in the LMD. Ideally, the findings from this project will be used to further tailor future nutrition and health interventions in the LMD to each of these populations in an effort to reduce health disparities and improve overall well-being.

\section{Background and Significance}

The LMD, a primarily rural region, has a large concentration of ethnic minorities, including Blacks and Latinos (University of Memphis Regional Economic Development Center [UMREDC], 2002; U.S. Census Bureau, 2013a). These ethnic populations, due in part to their low socioeconomic status (SES), suffer from nutrition-related health disparities more frequently than high SES or white populations (Fontenot et al., 2010; Zoellner, Connell, Bounds, Crook, \& Yadrick, 2009). Common inequalities experienced by minorities in this region include food insecurity, poor quality diets, overweight, obesity, and chronic disease. In order to help effectively reduce health disparities in these ethnic minority populations, interventions need to be culturally and regionally appropriate (Curry, 2000). Black culture in the LMD has been heavily influenced by the 
region's history of slavery (Ellison, 1995). Latino culture focuses primarily on the importance of family and interpersonal relationships (Galanti, 2003). In the LMD specifically, Latinos are affected by their history of immigration to the area as poor migrant workers (Rodriguez, 2012). Traditional diets, food availability, and the rich culture surrounding each of these population's dietary traditions are important to consider when planning appropriate nutrition and public health interventions.

PhotoVoice is a qualitative, community-based participatory research (CBPR) method that seeks to provide a "voice" to underserved populations by allowing project participants to take photographs of their community (Wang \& Burris, 1997). PhotoVoice projects in the realm of public health and nutrition can provide detailed insight into the health perceptions, including the perceived barriers and facilitators to health, of study participants (Martin, Garcia, \& Liepert, 2010). The use of PhotoVoice in this project will provide researchers a greater understanding of the health perceptions - and the similarities and differences in those perceptions - among Black and Latina women in the LMD, allowing these populations to be targeted more effectively for nutrition interventions in the future.

\section{Research Questions}

The purpose of this research is to assess the health perceptions among Black and Latina women in the Lower Mississippi Delta using visual depictions, journal reflections, and semi-structured interviews. From these findings, a conceptual framework will be developed to describe the health perceptions of minority women living in the LMD. The investigator seeks to answer the following research questions with this project: 
1. How do Black and Latina women in the LMD perceive health as individuals and for their communities?

2. What are the similarities and differences in health perceptions among Black and Latina women in the LMD?

3. How can these perceptions of health be utilized to promote change within minorities residing in this health disparate region?

\section{Theoretical Framework}

Grounded theory is a commonly used qualitative methodology that allows researchers to form a conceptual framework based on the findings of a project (Corbin \& Strauss, 1990). The resulting framework allows for the findings of qualitative studies to be generalized to a larger population, which is an uncommon yet desirable trait that legitimizes the research (Charmaz, 2000). In grounded theory, a detailed, multi-step analysis of the data collected from the study is performed in order to identify the most significant themes or categories. From these categories, the researcher will identify the "core category" — or the most prominent theme. This core category forms the foundation of the framework and seeks to explain the relationship between the core and other themes (Corbin \& Strauss, 1990). A model for combining grounded theory with PhotoVoice methodology in the context of public health was developed by Lopez, Eng, RandallDavid, and Robinson (2005) which will be utilized in this project. Using the PhotoVoice methodology, the researcher intends to assess the health perceptions of Black and Latina women living in the LMD. The findings from this grounded theory process will then be used to develop a theoretical framework that may have useful applications for researchers developing health interventions targeting ethnic minorities living in the LMD. 


\section{CHAPTER II}

\section{REVIEW OF RELATED LITERATURE}

The LMD region has a high concentration of health disparities that are likely related to the low SES of many of its residents (Everson, Maty, Lynch, \& Kaplan, 2002). The LMD region also has a large minority population—particularly Blacks, as well as a growing Latino population (UMREDC, 2002; U.S. Census Bureau, 2013a). Because of the power of tradition and cultural influence among minority groups, different strategies and interventions may be necessary to improve the health of these two populations (Curry, 2000).

PhotoVoice is a community-based participatory research (CBPR) method that was developed by Wang and Burris in 1997 to engage members of underserved communities in rural China (Strack, Lovelace, Jordan, \& Holmes, 2010). By equipping participants with cameras, researchers can identify common concerns that are shared by community members, as well as provide a structured way for community members to share these concerns with policymakers and key stakeholders within the community (Strack et al., 2010). The three main goals of PhotoVoice, as stated by Wang and Burris (1997), are "to record and reflect personal and community strengths and concerns; to promote critical dialogue and knowledge about personal and community issues through group discussion of photographs; and to reach policymakers" (p. 370). The PhotoVoice methodology has been used to help identify health perceptions of low-income minority communities through photographs, written reflections, and interviews (Catalani \& Minkler, 2010). PhotoVoice allows project participants to illustrate the barriers and facilitators to health in their communities, as well as propose solutions to reverse identified barriers (Wang \& 
Burris, 1997). The use of the PhotoVoice methodology has lead to a greater understanding of the factors that influence health and wellness in rural, low-income, minority communities, so interventions or policies can be developed to address vulnerabilities of the community (Catalani \& Minkler, 2010; Wang \& Burris, 1997).

It is important to consider the existing literature related to improving health among low-income, rural, minority populations. Much research has been conducted in the LMD addressing the region's high rates of poverty, food insecurity, poor diet quality, overweight and obesity, and chronic disease (Champagne et al., 2007; Fontenot et al., 2010; Smith et al., 1999; Stuff et al., 2004; Wang \& Beydoun, 2007). Although not related to the LMD specifically, many researchers have also developed methods for creating culturally appropriate interventions suited to a population's unique cultural background, traditions, and needs (Kreuter, Lukwago, Bucholtz, Clark, \& SandersThompson, 2002; Kumpfer, Alvarado, Smith, \& Bellamy, 2002; Marin, 1993; Parham \& Scarinci, 2007). Identifying perceptions of health, including perceived barriers and facilitators, is a key part of developing culturally appropriate interventions. Finally, research that has been conducted using the PhotoVoice methodology has allowed many common themes related to the health perceptions of residents of low-income minority communities to emerge for application in targeted nutrition interventions. This review of literature will cover the following topics: demographics and health disparities within the LMD; culturally appropriate interventions; and an overview of the PhotoVoice methodology and its applications in low-income, minority communities. 
The Lower Mississippi Delta

The LMD refers to the fertile areas surrounding the Mississippi River in Arkansas, Louisiana, and Mississippi. Many communities in the LMD formed due to the rich potential for agriculture in the area, and even today many residents of those communities work as farmers or in agriculture (Fontenot et al., 2010). Prior to the abolition of slavery, plantations in the LMD relied on African slave labor for planting, harvesting, and maintaining crops (Fontenot et al., 2010). After the abolition of slavery, when locally-owned farms began paying low wages to Black employees, the LMD thrived economically. Since the 1980s, a majority of farms operate as part of large corporations that use highly mechanized growing and harvesting techniques. After the industrialization of agriculture in this region, employment opportunities and equitable wages sharply decreased. Due in part to these drastic changes in agricultural practices, the LMD now has a high proportion of low-income, food insecure residents with a variety of chronic health problems that may be related to low socioeconomic status and lack of access to healthy foods (Connell et al., 2007; Fontenot et al., 2010).

According to Fontenot and colleagues (2010), 133 counties in Mississippi, Louisiana, and Arkansas are considered the historical "core" of the LMD; of these 133 counties, 126 have levels of poverty that are above the national average (Fontenot et al., 2010). The LMD region is largely rural, with few urban centers. Because of the LMD's agriculture base and lack of urban development, many of the LMD residents that are employed work in agriculture. Educational attainment among Delta residents is fairly low, and students' test scores in this region have trailed behind the rest of the country for decades (Jones, Thornell, \& Hamon, 1992; Mirvis, Steinberg, \& Brown, 2004). In a study 
on the causes of poverty in the LMD and the Texas Borderlands, researchers identified low educational attainment as one of the major contributors to poverty (Fontenot et al., 2010). The same study found that a high or increasing percentage of single-parent or female-headed households was a significant indicator of poverty, and the percentage of female-headed households in the LMD doubled between 1990 and 2000. The LMD also has a high population of minority residents, specifically Blacks (U.S. Census Bureau, 2013a). All of these factors are indicators of poverty and contributors to the relatively low socioeconomic status (SES) of LMD residents (Fontenot et al., 2010); because SES is negatively associated with body mass index (BMI) and health, these same factors may be contributing to the significant health disparities related to obesity present in the LMD (Wang \& Beydoun, 2007).

\section{Health Disparities}

The National Institutes of Health (2002) defines a "health disparate population" as one that has a higher concentration of health-related problems than the general population of the United States. Many minority groups in the United States are affected by these health disparities, some of which include high infant mortality, high rates of HIV infection, poor mental health, and high levels of nutrition-related preventable chronic diseases. The LMD has a high concentration of nutrition-related health disparities that are potentially related to the low SES of many of its residents (Everson et al., 2002). These disparities include a high number of food insecure households, poor diet quality of LMD residents, widespread prevalence of overweight and obesity, and a higher incidence and prevalence of chronic disease (Lower Mississippi Delta Nutrition Intervention Research 
Consortium [LMDNIRC], 2010; McCabe-Sellers et al., 2007; Mississippi State

Department of Health [MSDH], 2012a; Wang \& Beydoun, 2007).

Food insecurity and diet quality. Food insecurity, or lack of access to adequate or healthy food, has recently been identified as a significant problem in the LMD. In a study assessing the prevalence of food insecurity in the LMD, researchers found that $21 \%$ of households in the Delta were food insecure, compared to the national average of $14.5 \%$ (Coleman-Jensen, Nord, Andrews, \& Carlson, 2011; LMDNIRC, 2004). Researchers also identified that low-income Black households were the most likely to experience food insecurity with hunger, meaning that the members of the household frequently experienced the physical manifestations of hunger (LMDNIRC, 2004). However, food insecurity is not always accompanied by hunger; limited access to or limited consumption of healthy foods can also contribute to food insecurity (Champagne et al., 2007; Stuff et al., 2004). Champagne and colleagues (2007) found that food insecure adults living in the LMD had lower quality diets than food secure adults in the same region, as defined by how well each group was able to meet the Dietary Reference Intakes for selected nutrients. In a similar study, the Healthy Eating Index (HEI) was utilized with a sample of adults from the LMD; the study participants' scores were then compared to the average HEI scores collected from the National Health and Nutrition Examination Survey (NHANES), 1999-2000 (McCabe-Sellers et al., 2007). The researchers found that a higher percentage $(24.8 \%)$ of the study participants ate a diet defined as "poor" compared to the NHANES participants (18.3\%); the study sample also had a lower average HEI score (60.1) than the NHANES participants (63.4; McCabe-Sellers et al., 2007). McCabeSellers and colleagues (2007) concluded that the poor diet quality of LMD residents was 
related to low SES and lack of access to healthy foods like fruits, vegetables, and whole grains. These authors indicated that in order to increase the diet quality and food security of LMD residents, healthy foods must become more available and accessible; however other factors, like culture, appear to contribute to the poor diets in the LMD.

Access to healthy food is limited in the LMD compared to other parts of the United States. An abundance of research has been conducted on access to healthy food in the United States, and the general consensus is that rural, low-income, minority communities have greatly diminished access to healthy food compared to other communities (Connell et al., 2007; Farley et al., 2009; Larson, Story, \& Nelson, 2009). In a study in which the store shelf space of certain foods was compared in Louisiana, a rural state, and Los Angeles County in California, an urban county, the researchers found that stores in Louisiana had a more unhealthy "food environment" than stores in Los Angeles County and offered a higher percentage of unhealthy foods for sale (Farley et al., 2009). In a meta-analysis of 54 studies related to healthy food access, Larson and colleagues (2009) found that low-income and rural communities had more access to fast food restaurants while having less access to healthy foods when compared urban and higherincome areas. Larson and colleagues also found disparities related to ethnicity where minority communities had less access to fresh produce, healthy foods, and chain grocery stores compared to predominantly white or mixed-ethnicity neighborhoods. In a study that focused on the LMD region, researchers found that many Delta residents lived in extremely rural areas with no access to a grocery store (Connell et al., 2007). These residents were dependent on smaller food stores and convenience stores, which were 
limited in healthy offerings. Therefore, many residents of the LMD may depend on unhealthy convenience foods out of necessity.

Diet quality and food insecurity have also both been linked to health status in numerous research studies (Larson et al., 2009; Stuff et al., 2004). Limited access to supermarkets and easy access to convenience stores, which are both pervasive issues in low-income, rural, minority communities, have been linked to an increased risk for obesity, and researchers found that household food insecurity and self-reported health status were highly correlated (Stuff et al., 2004). In a sample of 1,163 food secure adults and 325 food insecure adults, only $16 \%$ of the food secure adults identified as having "fair" or "poor" health while $36.8 \%$ of the food insecure adults identified as having fair or poor health. Food insecurity and poor diet quality are significant issues facing lowincome LMD residents. Paradoxically, researchers have also found that food insecurity and hunger are risk factors for obesity (Dietz, 1995; Martin \& Ferris, 2007).

Overweight and obesity. In 2011, 34.6\% of Mississippi adults were considered obese (Centers for Disease Control and Prevention [CDC], 2012). The combined rate of both obesity and overweight in the state of Mississippi is 68.8\% (CDC, 2012). Ethnic minorities in Mississippi have the highest obesity rates, with $42.9 \%$ of Black and 35.4\% of Latino Mississippians categorized as obese (Kaiser Family Foundation, 2011; MSDH, 2012b). While Mississippi was the most obese state from 2004 to 2011, Louisiana currently has the highest obesity rate in the United States and Arkansas, another state with LMD residents, is currently the third most obese state. Residents of each of these states have self-reported obesity rates greater than or equal to $34.5 \%$ (CDC, 2013). It is difficult to determine if obesity and overweight are more concentrated the LMD regions 
of these states; however, an analysis of NHANES data by Wang and Beydoun (2007) revealed that obesity and overweight were more prevalent in rural, low-income, low-SES, minority communities. In this meta-regression analysis, researchers found that $70.7 \%$ of non-Hispanic Blacks and 72.5\% of Mexican Americans were overweight or obese. The researchers also found that children with low SES were more likely to be overweight than their higher SES counterparts. Therefore, it is likely that obesity and overweight are more concentrated in the LMD regions of Mississippi, Louisiana, and Arkansas where there is a significant low-SES, minority population.

Chronic disease. Overweight, obesity, and poor diet quality are significant indicators of nutrition-related chronic diseases such as type 2 diabetes, hypertension, hypercholesterolemia, heart disease, and certain types of cancer (Smith et al., 1999). Smith and colleagues (1999) compared the self-reported prevalence of nutrition-related chronic diseases in LMD counties and parishes, non-LMD counties and parishes (in Mississippi, Louisiana, and Arkansas), and other states. The LMD counties had higher obesity rates (31.3\%) than non-LMD counties (28.5\%) and other states $(25.1 \%)$. Additionally, the LMD counties had higher self-reported rates of diabetes, high blood pressure, and high cholesterol $(6.9 \%, 28.9 \%$, and $28 \%$, respectively) than non-LMD counties $(5.7 \%, 24.3 \%$, and $27.2 \%$, respectively) and other states $(4.8 \%, 21.3 \%$, and $27.4 \%$, respectively). The LMD counties also had more self-reports of "poor" or "fair" health status, and fewer residents had health insurance. As of 2012, 38.6\% of residents of LMD counties in the state of Mississippi had hypertension, $42.2 \%$ had hypercholesterolemia, and $12.1 \%$ had physician-diagnosed type 2 diabetes (MSDH, 
2012a). Overall, nutrition-related chronic disease (heart disease, stroke, cancer, and diabetes) caused approximately 55\% of deaths in Mississippi's Delta counties in 2010.

Health disparities associated with low SES are rampant in the LMD, a historically Black area with roots in agriculture (Fontenot et al., 2010). Low-income minority groups in the LMD, such as Blacks and Latinos, are disproportionately affected by poverty and health disparities (Zoellner et al., 2009). These health disparities include food insecurity, lack of access to healthy foods, high rates of overweight and obesity, and high rates of chronic disease. In order to effectively target these populations, interventions should be developed in a manner that is culturally sensitive and appropriate to the needs of these unique minority populations in the LMD.

Developing Culturally Appropriate Interventions

The LMD has a large concentration of racial minority populations. The Latino population in the LMD increased by $114.7 \%$ between 1990 and 2000, and Blacks comprise well over 70\% of many LMD counties (UMREDC, 2002; U.S. Census Bureau, 2013a). The southeastern United States, including the LMD, also has a significant and growing Latino population (Rodriguez, 2012). It has been noted in numerous research studies that minority populations tend to have low SES and poor health, which is particularly true for Black and Latino populations (Everson et al., 2002; Perez-Escamilla, 2009; Zoellner et al., 2009). The obesity rates of Blacks and Latinos are significantly above the national average, putting these groups at increased risk of nutrition-related chronic disease (Wang \& Beydoun, 2007). These groups are also more food insecure and tend to have poorer diets than white and Asian-American populations (American Dietetic Association [ADA], 2011; Haldeman, Gruber, \& Ingram, 2007). Therefore, it is 
important to develop culturally appropriate interventions that are unique to both Blacks and Latinos in order to effectively target and reverse the health disparities in the LMD (Curry, 2000).

In order to develop culturally appropriate interventions, it is essential to understand the unique health disparities that plague the population and the cultural influences and traditions that are prevalent. Specifically in public health and nutrition, it is important to consider cultural influences related to food, diet, exercise, and healthcare. According to Kumpfer and colleagues (2002), interventions that are "culturally-adapted" can improve outcomes for ethnic minority study participants and their communities. However, in order to be successful, culturally-adapted interventions should be unique to each target population based on ethnicity and geographic location, as opposed to a single intervention being developed for all ethnic minority groups (Kumpfer et al., 2002).

\section{Minority Populations}

Blacks account for $13.1 \%$ of the United States' population, making them one of the largest minority groups in the United States (U.S. Census Bureau, 2013b). As a historically oppressed minority group, Blacks - especially in rural areas like the LMDtypically have low SES, low educational attainment, and high rates of poverty (Fontenot et al., 2010; Mirvis et al., 2004). These factors may potentially put Blacks at increased risk for experiencing health disparities such as food insecurity, overweight and obesity, and chronic disease. In order to reverse these health disparities, targeted interventions should be developed based on the unique cultural needs of rural, low-income Blacks.

The southeastern United States has been dependent on Black farm labor since preCivil War times when slavery was a legal and common practice (Rodriguez, 2012). 
Therefore, slavery has heavily influenced the cultural practices and religious traditions of Black Americans. Religious involvement, particularly in Judeo-Christian churches, is very important to many Black communities in the southeastern United States (Ellison, 1995). As a whole, Blacks tend to be more involved in church and religious activities than whites, and researchers have even suggested that religion plays an important role in improving and maintaining mental health of the individuals in these communities (Ellison, 1995). Black churches in the South tend to be important facets of the community, allowing community members to come together for worship, socialization, and community service; Black churches also serve as places where residents can present their concerns and problems related to the community and find solutions (Ross \& Wheeler, 1971). The importance of church, community, and family in Southern Black culture may have developed during the era of slavery as a way for Black slaves to cope with their difficult life situations and gain valuable social support from their peers (Nelsen, Yokley, \& Nelsen, 1971).

The modern diet of rural, low-income Blacks living in the South has also been shaped heavily by the past, including ties to African ancestry and cultural relics from the era of slavery (Airhihenbuwa et al., 1996). Airhihenbuwa and colleagues (1996) found that older Blacks associated their food choices with the traditional food choices made by their ancestors throughout periods of slavery and discrimination, while younger Blacks made their food choices based on taste and personal preference. These researchers also found that the concept of "soul food" was very important to the cultural identity of the study participants. While many of the participants expressed that some aspects of traditional soul food were unhealthy, all the participants indicated that it was very 
important for soul food to continue on as a unique cultural food tradition among blacks (Airhihenbuwa et al., 1996). Unfortunately, the availability of the fresh, local produce and meats - considered such an integral part of soul food — has been limited in areas like the LMD (Airhihenbuwa et al., 1996; Connell et al., 2007). As a result of this limitation, many low-income Blacks living in the LMD are dependent upon packaged convenience foods and fast food meals because these foods are inexpensive and widely available (Connell et al., 2007). Important aspects of Southern Black culture such as spirituality, soul food, and food availability should be incorporated into interventions targeted at lowincome, rural Black populations in order to effectively reduce nutrition related health disparities in these communities.

Researchers studying the Black population in the LMD have found that successful interventions share some specific characteristics. Blacks living in the LMD respond positively to nutrition interventions that are culturally appropriate and that allow for social interaction and involvement with family and other community members, incorporating the ideals of community service (Parham \& Scarinci, 2007). Churches are often successful venues for nutrition interventions aimed at reducing health disparities in Black communities. Zoellner and colleagues (2009) found that Blacks in the LMD do not regularly use computers or the Internet and that most residents of the LMD get nutrition information from television, health professionals, or other people in the community. Researchers have found that children and families in low SES areas have limited access to home computers and internet access when compared to children in high SES areas (Thomas, 2008). When developing interventions targeted at Blacks in the LMD, these factors should be carefully considered and woven into the program. However, the LMD 
also has a significant and growing Latino population, and it is important to consider the unique cultural needs of this population as well (UMREDC, 2002).

Latinos are now the largest minority group in the United States, accounting for $16.9 \%$ of the entire population (U.S. Census Bureau, 2013b). Latino populations, many of which are low in SES, experience disparities similar to those of Blacks, including food insecurity, overweight and obesity, and chronic disease. Multiple researchers have found that Latino immigrants living in rural areas experience food insecurity with and without hunger at much higher rates than urban Latino families and other ethnic groups (Haldeman et al., 2007; Quandt et al., 2006). Acculturation to life in the United States also appears to be a significant contributor to health disparities in Latino populations. Among Latino immigrants, acculturation often results in adoption of a "standard American diet" high in calories, fat, and refined carbohydrates (Perez-Escamilla, 2009). Culturally appropriate interventions should address English and non-English speaking Latinos, as well as provide resources and support for Latino families and communities looking to preserve their traditional, healthy eating patterns (Perez-Escamilla, 2009).

Traditional Latino and Hispanic cultures are highly divergent from the majority culture of the United States. Family is highly regarded in Latino culture where extended family homes are common in Hispanic countries, and many Latino children often live at home with family until marriage (Galanti, 2003). The concept of familismo, or unity with one's family, is very important in traditional Hispanic cultures. The family, as opposed to the individual, is the unit of choice for decision making. Traditional gender roles are common among Latino families, including first-generation immigrants to the United States. The male is often in the provider role, while the female usually remains home to 
maintain the household, cook, and care for the children. As the level of acculturation increases, these traditional cultural influences may not play as large a role on Latino immigrants' behaviors and attitudes (Perez-Escamilla, 2009). Personalismo is an important type of relationship with non-relatives, defined as a strong and trusting friendship; for example, traditional Latinos may disregard health advice from a professional until the professional has established personalismo with them (Galanti, 2003). Because Hispanic and Latino immigrants come from such a large variety of countries and cultures, not all Latinos will ascribe to familismo, traditional gender roles, and personalismo (Galanti, 2003). Traditional Hispanic and Latino culture also influences the way Hispanics and Latinos living in the United States prepare and eat food (Castellanos, Connell, \& Lee, 2011; Perez-Escamilla, 2009).

The traditional Latino diet is one that is high in fruits and vegetables and low in sodium, added fat, and refined sugar (Kittler \& Sucher, 1998). Overall, this traditional diet is healthy and balanced. However, due to a variety of factors, many Latinos living in the United States have limited access to healthy foods. Latinos in rural areas are much more prone to experiencing food insecurity than their urban counterparts (Haldeman et al., 2007) because many rural areas do not have grocery stores; instead, residents must purchase food from convenience stores or fast food restaurants, which are limited in selection and offer fewer healthy options like fresh produce (Connell et al., 2007). Even if healthy options are available, many Latinos will begin to consume a "Western" diet full of processed, packaged foods; fast food; and added sugar and fat as they become accustomed to living in the United States (McArthur, Anguiano, \& Nocetti, 2001). Latinos born in the United States also express a preference for American foods like 
hamburgers and French fries, which are not as healthy as traditional Latino foods. The importance of family, the level of acculturation, and the traditional Latino diet are extremely important considerations when developing nutrition interventions targeted at Latinos.

Successful public health interventions aimed at Latinos should be based on Hispanic/Latino cultural values, composed of activities and strategies that are appropriate to the culture, and reflective of the target population's preferences (Marin, 1993). Specifically, Latinos are interested in maintaining the integrity of their native language, so interventions should be available in Spanish or an interpreter should be readily available (Marin, 1993). Many Latinos often view health and disease in a very holistic way, rooted in long-term behaviors as well as family relationships (Landrine \& Klonoff, 1992). Simpatía, a concept similar to familismo and personalismo, is a way to define the importance of interpersonal relationships in Latino culture; interventions that are longterm or family-based are successful among Latino populations because they foster simpatía (Triandis, Marin, Lisansky, \& Betancourt, 1984). Culturally appropriate interventions should consider levels of acculturation, cultural traditions, family, and interpersonal relationships in order to successfully reduce health disparities in Latino populations.

Perceptions of health and cultural needs can also vary significantly from community to community (Kreuter et al., 2002). It is important to consider the unique needs of a target population based on both its cultural composition and its geographic location (Kreuter et al., 2002; Kumpfer et al., 2002). For example, the LMD is a primarily rural and low-income area, meaning that the Blacks and the Latinos living in 
the LMD will have different health needs and perceptions than those residing in urban or high-income areas (Fontenot et al., 2010; Kumpfer et al., 2002). One unique qualitative methodology than can be used to identify the perceived needs and health-based ideas of a particular population is PhotoVoice, a type of CBPR method (Wang, 1999).

\section{PhotoVoice}

PhotoVoice methodology was first used to assess the health perceptions of rural, low-income women living in Yunnan Province, China (Wang, Yi, Tao, \& Carovano, 1998). The methodology, which utilizes photographs to form a narrative based on each participant's experiences, was developed to provide a "voice" to underserved communities using photographs (Wang et al., 1998). The methodology is rooted in critical feminist theory, as it is focused on the perceptions and experiences of historically oppressed groups such as women and ethnic minority groups (Wang et al., 1998). Therefore, PhotoVoice is uniquely suited for use in low-income minority communities such as the LMD.

\section{Elements of PhotoVoice Projects}

Although a framework for PhotoVoice projects has been developed, the methodology is easily customizable to meet the needs of the target community and program participants (Catalani \& Minkler, 2010; Wang, 1999). According to Wang (1999), one of the original developers of the PhotoVoice methodology, there are five key concepts that should be incorporated in PhotoVoice projects: (1) images can be used as teaching tools; (2) photographs have the power to influence policy; (3) community members should be involved in creating and defining health-related public policy; (4) policymakers and key stakeholders should serve as an audience for community members; 
and (5) an emphasis on individual and community action. These key concepts allow PhotoVoice projects to serve a dual purpose of gaining participant perspectives while serving as a catalyst for positive community change (Wang, 1999).

In conducting PhotoVoice research, 10 steps have been described and include identification of a target community, recruitment of participants, training of participants, providing a set period of time for participants to take photographs, meeting with participants to discuss photographs, and dissemination of photographs and stories to policymakers and community leaders (Wang, 1999). In a review of literature on PhotoVoice methodology, Catalani and Minkler (2010) identified four processes that were universally present in PhotoVoice projects: partnership building and community participation, training, research and documentation, and photo-elicited discussion. These guidelines and processes can be used to develop PhotoVoice projects that yield powerful information from and about the target population. Moreover, Wang's (1999) suggestions can also be used to help formulate innovative, thoughtful, and well-planned projects in the realm of public health and nutrition.

\section{PhotoVoice Methodology Applications}

PhotoVoice methodology has a variety of applications within the fields of public health and nutrition (Martin et al., 2010). Because it is a CBPR method, PhotoVoice utilizes participants as a research instrument and allows participants to be actively involved in the research process (Wang et al., 1998). PhotoVoice can be used in a variety of populations, regardless of ethnicity, gender, age, education, or literacy level (Martin et al., 2010). In one meta-analysis of PhotoVoice projects in nutrition and dietetics, the researchers concluded that PhotoVoice could be successfully implemented in both 
clinical and community settings for a variety of purposes: to serve as a qualitative "food record" in clinical or school settings; to illustrate challenges related to diet, disease state, and budget in young people, the elderly, and single-parent households; and to identify the barriers and facilitators to healthy eating in a variety of populations (Martin et al., 2010).

Because the PhotoVoice methodology was originally developed to give a voice to underserved peoples, it has many useful applications (Catalani \& Minkler, 2010; Martin et al., 2010). It is often used as a tool to investigate the barriers and facilitators to physical activity and healthy eating that exist within certain communities, such as low-income rural towns or inner city neighborhoods (Neill, Leipert, Garcia, \& Kloseck, 2011; Valera, Gallin, Schuk, \& Davis, 2009). The methodology can also be applied in order to understand the perspective of certain very specific populations such as low-income, immigrant, Latina mothers (Torres, Meetze, \& Smithwick-Leone, 2013). For example, this study assessed the perceptions of childhood obesity in a rural area from the point-ofview of immigrant Latina mothers with school-aged children. These mothers identified barriers and facilitators to physical activity for their children and also proposed solutions to their perceived barriers. In many other PhotoVoice projects, the participants proposed solutions to overcoming perceived barriers in addition to identifying barriers. Because one of the primary goals of PhotoVoice is to facilitate positive change and influence public policy in the community, researchers should seek to provide solutions to the barriers identified in these projects (Wang, 1999).

Barriers to health. In many PhotoVoice projects about health, participants were asked to photograph perceived barriers to healthy eating and exercise within the community. Interestingly, many common themes emerged despite the diversity of the 
projects. In a project on barriers to physical activity in Latina teens, the participants identified rusted-out playground equipment, high-traffic roads, and lack of sidewalks as significant barriers (Hannay, Dudley, Milan, \& Leibovitz, 2013). In a similar project about the effect of the rural environment on children, participants noted that high traffic areas and few sidewalks were barriers to physical activity (Findholt, Michael, Davis, \& Brogoitti, 2010). Lack of safe playground equipment and sidewalks were also cited as barriers in other PhotoVoice programs (Torres et al., 2013; Wang \& Pies, 2004). Other oft-cited barriers to physical activity included: lack of transportation to and from recreation areas, lack of time, limited availability of recreation programs, and discrimination or cultural barriers (Findholt et al., 2010; Hannay et al., 2013; Torres et al., 2013).

There were also a number of frequently reported barriers to healthy eating. In a PhotoVoice project about weight loss motivation in obese adolescents, participants took photos of sodas and fast food meals to represent the most significant barriers to healthy eating (Woolford et al., 2012). Similarly, Findholt and colleagues (2010) found that participants perceived the ease and affordability of obtaining unhealthy food as a barrier to eating healthy; these participants photographed candy displays in grocery stores, banners advertising sales on large bags of candy and large sodas, and placement of sugary cereals on grocery store shelves at eye level with young children. The participants in this project also identified lack of time as a barrier to healthy eating; one participant noted that it is "just easier to go to McDonald's" than to prepare meals at home. In one PhotoVoice project with at-risk youth who were enrolled in a healthy cooking program, the participants identified the two most difficult barriers to overcome as ease of access to 
both fast food restaurants and unhealthy food at home or in stores (Thomas \& Irwin, 2013). To illustrate this point, one participant took a photograph of a fast food drivethrough sign advertising that the restaurant was open twenty-four hours a day.

In a PhotoVoice project about women's access to healthy food in Harlem, the participants were primarily unemployed, low-income minority mothers; these women identified the expense of healthy food as one of the most significant barriers to healthy eating. Although the women could identify healthy foods, it was easier to stay within budget by purchasing inexpensive, unhealthy foods (Valera et al., 2009). Many participants took photographs of expensive healthy foods in the grocery store like salmon and vegetables to illustrate the financial burden that purchasing such foods would cause (Valera et al., 2009). In another PhotoVoice project investigating food insecurity among female-headed households, the participants identified a number of barriers to food access including discrimination, lack of support from the fathers of their children, unavailability of jobs, and dependency on the welfare system (Chilton, Rabinowich, Council, \& Breaux, 2009). Older rural women using PhotoVoice identified the following barriers to food access and preparation: physical disability, changing family size, winter weather conditions, inability to read food labels, and lack of resources (Neill et al., 2011). One participant took a picture of a snow drift that completely blocked her driveway, piled up almost to the top of her mail box. This photo illustrated that winter weather made travel to the grocery store unsafe and difficult. Although there appear to be many barriers to healthy eating and physical activity, it is important to remember that the barriers to health can vary widely depending on the SES, location, resources, and ethnic makeup of the 
community. Many participants in PhotoVoice programs were able to identify facilitators to health as well.

Facilitators to health. Facilitators are certain elements within the community that positively contribute to healthy eating and physical activity among study participants and community members (Neill et al., 2011). In a PhotoVoice project on the effects of a rural environment on children, participants cited the natural environment and the prevalence of organized sports as facilitators to physical activity (Findholt et al., 2010). Participants took photographs of a lake where children often congregate to play, as well as an outdoor basketball court in the community. In a similar PhotoVoice project on obese adolescents, a participant took a photo of a clear sky and stated that good weather is a facilitator to physical activity (Woolford et al., 2012). In another project based in rural Oregon, study participants cited the community's collective focus on local agriculture and gardening as a facilitator to healthy eating (Findholt et al., 2010). Youth enrolled in a cooking program reported that the cooking program itself was a significant facilitator to healthy eating, as it increased their "food literacy," or their general knowledge of food selection, preparation, storage, and nutrition. One participant took a picture of a whole chicken before and after, portioning it to illustrate newfound knowledge. Another participant was photographed in a field of beans in front of three barrels overflowing with her picks (Thomas \& Irwin, 2013). Rural older women reported the following facilitators to food access: gardening, food stockpiling, social support, and experience with cooking. One participant photographed her stockpile of canned vegetables, seasonings, and sauces (Neill et al., 2011). While these projects illustrated many facilitators to health, the 
barriers appear to outweigh these facilitators (Findholt et al., 2010; Torres et al., 2013; Valera et al., 2009).

Solutions to identified barriers. In many PhotoVoice studies, the participants proposed solutions to overcoming barriers to physical activity and healthy eating within the community. In a PhotoVoice project that assessed the perspectives of obese adolescents, participants agreed that the photographs taken during the study would serve as motivational reminders to be healthy if the photographs were sent to participants via text message at a later date (Woolford et al., 2012). Similarly, in a project in which a Latina teen took a photo of a community pool that had been shut down, citing this as a barrier to physical activity within the community, the participants were able to collect over 100 signatures on a petition to have the pool reopened (Hannay et al., 2013). The participants also suggested an afterschool alternative to physical education classes, which has now been implemented at a local community center. Latina mothers asked to identify factors contributing to childhood obesity in the community proposed a number of solutions that would serve to increase physical activity and healthy eating: modeling healthy eating to children; home and community gardening; and organizing a meeting with key stakeholders within the schools, the neighborhoods, and the city to petition for more police presence in the area (Torres et al., 2013). In one project assessing lowincome women's access to healthy food in Harlem, one woman took a photo of a plate of homemade dumplings and explained that her solution to high food prices was to make meals from scratch, which was both inexpensive and healthy (Valera et al., 2009). Other women in the study stated that shopping outside of Harlem was a way to have access to wider varieties of inexpensive fresh fruits and vegetables. In many of these projects, the 
solutions to barriers to health were simple, inexpensive, and beneficial to the community as a whole. PhotoVoice not only allows participants to identify barriers and facilitators to health within the community, it also allows participants to advocate for positive change and become a driving force for improving the health of the community.

PhotoVoice Methodology Strengths and Limitations

The PhotoVoice methodology has a number of strengths. As a participatory action research method, it can be used as a tool to facilitate a dialogue between community members and community leaders (Strack et al., 2010). Merely equipping participants with cameras allows the study participants to advocate for positive change within the community by highlighting areas of concern, which results in a more complete understanding of the community's needs, as well as the community's strengths (Catalani \& Minkler, 2010). PhotoVoice also empowers individuals within the community by providing opportunities for individual citizens to influence community leaders. Wang and colleagues (1998) outlined a number of potential advantages to all participants in a PhotoVoice project, including positive feelings related to contributing to changes in the community, improving the quality of life for community members, and gaining increased credibility through collaboration with key stakeholders. However, some community members have more power and influence than others. Therefore, the participants with more power are able to learn from others in the community, become innovators, and form closer ties with the community as a whole (Wang et al., 1998). Those in the community with less power are able to increase access to power and participate in improving the community through taking pictures and story-telling (Wang et al., 1998). 
However, there are also limitations to using the PhotoVoice methodology. The safety of participants is a very important concern. Participants may put themselves at risk for harm by trying to capture an image from a certain vantage point or by photographing people who do not wish to be photographed (Wang et al., 1998). Because participants may be asked to take pictures of negative aspects of the community, the project may be polarizing or controversial. Participants should take care to be sensitive to the feelings of other community members. However, community pressure may force the participant to censor him- or herself by avoiding photographing the subjects that are truly problematic within the community. Photographs are also difficult to analyze, as it can be difficult to extract the participant's intended meaning from the photographs and participant accounts. Furthermore, the PhotoVoice methodology can also be expensive, as each participant must be provided with a camera, film or memory, and training.

The PhotoVoice methodology is a useful tool for examining health perceptions within communities (Yeh et al., 2008). Findings from PhotoVoice projects can be used to help shape future research of the target population by further customizing future interventions to meet the needs of the population. Identification of perceived barriers and facilitators to health is vital to tailoring interventions to specific communities (Yeh et al., 2008). As a CBPR method, PhotoVoice empowers individuals and communities to take charge of their health and become advocates for positive and healthy changes within the community (Wang, 1999).

\section{Grounded Theory}

Grounded theory is a methodology that can be applied to qualitative research to lend generalizability to the findings (Charmaz, 2000). In grounded theory studies, the 
researchers develop a conceptual framework based on the study findings (Corbin \& Strauss, 1990). This conceptual framework is meant to summarize and explain a phenomenon or paradigm, and ideally will be applicable to populations that are similar to the study population. A well-executed data analysis is vitally important to successful grounded theory studies (Creswell, 2005). In most grounded theory studies, a three step coding process is used in order to develop a conceptual framework: (1) open coding, or the preliminary division of data into related themes or categories; (2) axial coding, or the development of a coding paradigm which describes relationships between the categories; and (3) selective coding, or the refinement of the coding paradigm into the finalized conceptual framework. These three steps are discussed more thoroughly in Chapter III: Methodology. Grounded theory has been extensively used in qualitative health research; however, few researchers have combined grounded theory with PhotoVoice methodology (Lopez et al., 2005).

Lopez and colleagues (2005) completed a novel study using grounded theory and PhotoVoice methodology that aimed to explain the coping mechanism and quality-of-life concerns of rural Black breast cancer survivors. The conceptual framework that resulted from the researchers' findings described the relationships between social forces, qualityof-life concerns, and coping mechanisms of the participants. "Spiritual faith" and "maintaining social standing" were identified as the primary methods of maintaining quality-of-life for these participants. In addition to this conceptual framework, Lopez and colleagues developed a model for combining grounded theory and PhotoVoice methodology. This model is illustrated and described in more detail in Chapter III: Methodology. In a similar study, researchers utilized PhotoVoice and grounded theory in 
order to examine, analyze, and describe the factors that contributed to dietary acculturation and eating habits of Hispanic males (Castellanos et al., 2013). These researchers found that there were many factors that "enabled" poor dietary habits in this population, including time constraints, inadequate nutrition knowledge and cooking skills, and limited access to healthier options. From these findings, a grounded theorybased conceptual framework utilizing the "precede-proceed" model was developed to describe factors that shaped the dietary patterns of traditional Latino males in the United States. In combination with PhotoVoice methodology, grounded theory allows researchers to elicit rich, detailed information from participants in order to develop a conceptual framework that accurately captures the paradigm being studied.

\section{Conclusion}

The LMD, a primarily rural area, has a high concentration of low income, low SES, ethnic minority residents (Fontenot et al., 2010). These disadvantaged communities, particularly Black and Latino populations, have a great deal of health disparities compared to other communities in the United States (ADA, 2011). The health disparities these communities face include high levels of food insecurity, poor quality diets, overweight and obesity, and nutrition-related chronic disease (Kaiser Family Foundation, 2011; LMDNIRC, 2004; McCabe-Sellers et al., 2007; Smith et al., 2009). However, because of the unique ethnic minority composition of these communities, culturally appropriate approaches and interventions are absolutely necessary. Blacks and Latinos have unique cultures, traditions, and needs that must be addressed when attempting to decrease the health disparities that plague these populations (ADA, 2011; Curry, 2000). 
PhotoVoice is a useful way to gain the perspective of individuals in underserved communities (Wang \& Burris, 1997). Equipping study participants with a camera to take photographs allows participants to honestly assess the state of the community and identify its strengths and weaknesses (Catalani \& Minkler, 2010). The PhotoVoice methodology is easily tailored to meet the needs of many populations and communities. PhotoVoice can empower the community and allow participants to serve as catalysts for positive change (Catalani \& Minkler, 2010). Many communities have a number of barriers to health in common: unsafe streets and play areas, lack of transportation, few recreational programs, lack of time, discrimination, and the prohibitive expense of healthy food versus the inexpensiveness and ease of unhealthy food (Findholt et al., 2010; Hannay et al., 2013; Torres et al., 2013; Valera et al., 2009; Wang \& Pies, 2004; Woolford et al., 2012). However, PhotoVoice participants in these same communities were also able to identify factors in the community that had a positive effect on health: organized sports, the natural environment, and community gardening (Findholt et al., 2010; Valera et al., 2009; Woolford et al., 2012). The PhotoVoice process also allowed participants to propose solutions to overcoming health barriers to community leaders, resulting in positive changes within the community (Hannay et al., 2013; Torres et al., 2013). PhotoVoice allows participants to positively impact the health of the community through individual empowerment, increased understanding of community strengths and weaknesses, and outreach to policymakers and key stakeholders (Catalani \& Minkler, 2010). The use of grounded theory allows researchers to develop conceptual frameworks based on the study findings, which lends generalizability to qualitative research (Charmaz, 2000). In prior studies, the combination of grounded theory and PhotoVoice 
methodology has been successfully applied to rural, low-income, minority populations (Lopez et al., 2005). Grounded theory and PhotoVoice methodology may be excellent tools for exploring the unique needs of rural, low-income, minority populations in the LMD - in order to strengthen future interventions aimed at decreasing the health disparities that plague the region. 


\section{CHAPTER III}

\section{METHODOLOGY}

\section{Research Design}

This grounded theory research utilized PhotoVoice, a qualitative CBPR method developed for use with underserved populations (Wang \& Burris, 1997). In PhotoVoice projects, study participants are given a camera and asked to take photographs that relate to the topic being researched. Participants may also be asked to write journal reflections and participate in interviews or focus groups about their photographs. The data collected from any PhotoVoice project consists of photographs and words that form a participant's "narrative" about the study topic. In this study, these narratives were studied and coded for significant themes. After analysis, the findings of this study were used to develop a grounded theory-based framework to describe the health perceptions of minority women living in the LMD.

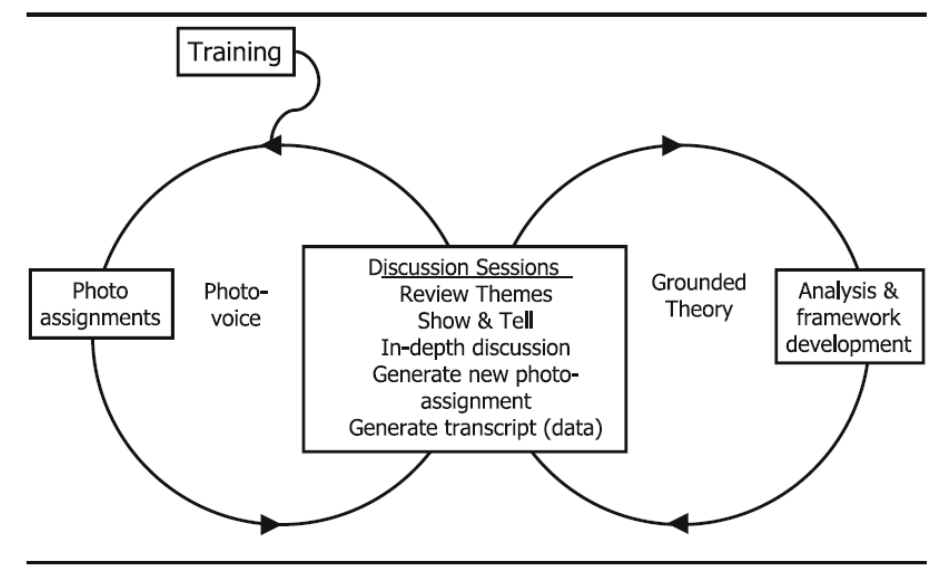

Figure 1. A model for merged PhotoVoice and grounded theory projects. Adapted from "Quality-of-Life Concerns of African American Breast Cancer Survivors Within Rural North Carolina: Blending the Techniques of PhotoVoice and Grounded Theory," by E. D. S. Lopez, E. Eng, E. Randall-David, and N. Robinson, 2005, Qualitative Health Research, 15, p. 105. Copyright 2005 by Sage Publishing. Reprinted with permission. 
The design of the current project is based on Figure 1, which illustrates a combination grounded theory-PhotoVoice model developed by Lopez and colleagues (2005). According to this model, the following steps should be followed in combination grounded theory-PhotoVoice projects:

Step 1. The first step in project facilitation is training and orientation, in which participants are familiarized with the purpose of the project and given directions on camera use. Participant training is an important component of all PhotoVoice projects (Wang, 1999).

Step 2. The next step in the project consists of participants taking photographs in their communities and making journal reflections using guiding questions about their photographs.

Step 3. PhotoVoice and grounded theory intersect when the researchers and the participants meet to discuss the photographs. It is at this point that data analysis begins, which is an important facet of both grounded theory and PhotoVoice methodology.

Step 4. After the discussions are completed, a more comprehensive data analysis begins. Data sources will include photographs, journal reflections, transcripts, and existing research to develop a grounded theory-based framework that summarizes and explains the major findings of the project.

\section{Participants}

The participants in this project were recruited through a two-step combination of purposive sampling and snowball sampling. To continue the theme of women's passion for "the health of the Delta" (Huye et al., 2014, p. 41), the researchers sent invitations to participate to women who participated in the Mississippi Communities for Healthy 
Living (MCHL) nutrition intervention formative research phase. A mailed letter was sent to these former participants $(n=8)$ requesting their participation in the current project. Minority women who were already active members of community organizations or those who were actively involved in health promotion within the community were recruited for participation as well. Another venue for recruitment was a health fair that took place in the LMD sponsored by MCHL. The snowball sampling component included recruitment of Latina participants from one Latina participant who is very involved in health promotion in the Latino community in the LMD. This project has been approved by The University of Southern Mississippi’s Institutional Review Board.

\section{Data Collection}

\section{Initial Meeting}

The initial meeting included informed consent, participant training and orientation to the project, a brief preliminary interview, and completion of a demographic questionnaire. Prior to beginning data collection, informed consent was obtained from each participant (Appendix A). Participant information packets were provided to each individual (Appendix B). During the training component, the researchers shared how the project was developed, the scope of the project, basics of the PhotoVoice methodology, and how to operate a digital camera. Because the "stories" that accompany the photographs are a very important component of the research, participants were also prompted to record thoughts and observations at the time of picture-taking (Catalani \& Minkler, 2010). The participants were asked to consider the following questions when taking their photographs:

- What activities best show health or a healthy lifestyle in my community? 
-What activities reflect the opposite of a healthy lifestyle in my community?

-What in my life or community has helped me live a healthy lifestyle?

- What in my life or community has prevented me from living a healthy lifestyle?

- What do I want to tell other people about living a healthy lifestyle?

- What are my hopes for the future? And what might help me get there?

The participants were also asked a series of introductory interview questions about their current perceptions of health in the LMD. To prompt discussion, the interviewer read the participants some relevant quotes from a prior focus group. The introductory interview questions were:

- What do you think about when you hear quotes such as these? What do you think about the health of the Delta or your community?

- What would you like to change about your health or the health environment of your community?

- If you had to draw a picture of the health of your community or for you personally, what would it look like?

Each participant also completed a demographic questionnaire prior to the end of this initial meeting (Appendix C). At the close of this meeting, participants received a digital camera, a memory card, and a journal. The interview portion of each initial meeting was digitally audio-recorded, transcribed, and included in data analysis.

\section{Photo-Taking Period}

Each participant was given a two- to four-week period in which to take her photographs and record journal reflections. Participants were provided a self-addressed, stamped envelope in which to the return the camera's memory card and journal to the 
researcher. The participants also had the option to email electronic versions of the photographs and journals to the researcher. The researcher reviewed each set of photographs and journal entries and developed semi-structured interview guides for each participant based on the content of the participant's photographs and journal. All photographs and journal entries received from participants were included in data analysis. The participants were then invited to the second and final interview.

\section{Final Meeting}

The second interview was part of a process which is unique to the PhotoVoice methodology, called "participatory analysis." When the photo-taking portion of the project was over, participatory analysis began. It involved the researchers and the participants coming together to discuss and analyze the photographs. Participatory analysis includes selection of photographs to discuss, structured interviewing and participant storytelling, identifying common issues and themes in the photographs, and recording the findings (Wang et al., 1998). Wang suggests using the "SHOWeD" method of interviewing in order to glean the most pertinent details about a photograph from the study participants (Wang et al., 1998). The SHOWeD method is a series of questions:

- What do you see here?

- What's really happening here?

- How does this relate to our lives?

- Why does this problem or this strength exist?

- What can we do about this? (p. 80)

The goal of the second interview was to include the participants in the preliminary steps of data analysis, as well as to collect more information from each participant about 
the subject matter of their photos and journal entries. Each of these final interviews was digitally audio-recorded, transcribed, and included in data analysis.

\section{Data Analysis}

The four primary sources of data resulting from this project were photographs, journal entries, interviewer notes, and interview transcripts. Demographic data were entered into Microsoft Excel and analyzed for measures of central tendency and descriptive statistics. Photographs, journal entries, field notes, and interview transcripts were analyzed using an established qualitative method called continuous content analysis, and during this step, categories and subcategories within the data were identified. Next, axial coding and selective coding were completed and helped develop the grounded theory described below. Open coding was done for each participant's individual narrative, and then for the data as a whole. Separate continuous content analyses were also performed on data collected from Black participants and from Latina participants. This process resulted in a set of themes that fully described the data and that reflected the differences and similarities in health perceptions among Black and Latina women in the LMD. Collaborative analysis with a second coder ensured reliability of the themes that were identified. Peer debriefing was also performed to ensure that the codes are accurate and reliable. This peer debriefing occurred with a colleague who has lived in the LMD and has experience in similar research activities that have taken place in the LMD. After open coding was completed, the framework development process began.

\section{Framework Development}

Throughout the process of data collection in a grounded theory project, the researcher must consider the potential for conceptual theory development. In a novel 
PhotoVoice-grounded theory combination project by Lopez and colleagues (2005), researchers developed a framework to describe the quality of life of Black breast cancer survivors from a rural area. The researchers completed multiple cycles of the model outlined in Figure 1 to fully incorporate the photographs and the meaning expressed in the photographs into the framework that was eventually developed from the project. The current project will use the same model to guide the development of the framework based on the tenets of both PhotoVoice methodology and grounded theory. Table 1 provides definitions of the coding terminology used in this study.

Table 1.

Coding Terminology and Definitions

\begin{tabular}{ll}
$\begin{array}{l}\text { Term } \\
\text { Continuous content } \\
\text { analysis }\end{array}$ & $\begin{array}{l}\text { Cofinition } \\
\text { Continuous identification of categories and subcategories } \\
\text { process (Krueger, 2013) }\end{array}$ \\
\hline Open coding & $\begin{array}{l}\text { Categorization of data into broad themes and categories } \\
\text { (Corbin \& Strauss, 1990) }\end{array}$ \\
\hline Axial coding & $\begin{array}{l}\text { The process of describing the relationships between categories } \\
\text { (Creswell, 2005) }\end{array}$ \\
\hline Core category & $\begin{array}{l}\text { The category or theme that appeared to be the most prevalent } \\
\text { or significant to participants (Corbin \& Strauss, 1990) }\end{array}$ \\
\hline Coding paradigm & $\begin{array}{l}\text { A diagram illustrating the relationship between the core } \\
\text { category and other categories in the context of the study } \\
\text { purpose (Corbin \& Strauss, 1990) }\end{array}$ \\
\hline Selective coding & $\begin{array}{l}\text { The process of refining the coding paradigm into the final } \\
\text { product: a grounded theory-based conceptual framework } \\
\text { (Corbin \& Strauss, 1990; Creswell, 2005) }\end{array}$ \\
\hline
\end{tabular}

After open coding was completed and data were organized into themes and subthemes, axial coding began. First, the core category was identified. Next, the relationship of the core category to the secondary categories, with attention to how all of the sub- 
categories fit, was determined. The result was a coding paradigm. Selective coding was then conducted. From the coding paradigm developed during the axial coding process, the researcher refined the framework based on the data collected during the study, existing research, and input from colleagues (Corbin \& Strauss, 1990). At this step, the researcher chose the most appropriate way to diagram the framework of health perceptions among minority women in the LMD (Creswell, 2005). During open coding, the perceptions of Black and Latina participants appeared to be highly divergent, so the researcher performed axial and selective coding on data collected from Black participants and Latina participants separately. This process allowed the researchers to illustrate the major health perceptions among these minority groups, as well as the general paradigm of health perceptions of minority women in the LMD by developing a framework. Ideally, the conceptual framework that arose from this combination grounded theory-PhotoVoice research would allow researchers to develop more culturally appropriate interventions for Black and Latino populations in the LMD and thus decrease health disparities within these populations. 


\section{CHAPTER IV}

\section{RESEARCH FINDINGS}

Demographic Information

Ten women were recruited for participation in the study, and only one participant dropped out of the study prior to its completion. This participant's demographic data has been excluded from analyses. All participants were females between the ages of 18 and 70 years old. Two participants identified as Hispanic/Latino, and the remaining seven participants identified as Black. Demographic data are detailed in Table 2.

Table 2

Demographic Data $(N=9)$

\begin{tabular}{|c|c|c|c|}
\hline & Latinas $(n=2)$ & Black Women $(n=7)$ & Total $(\mathrm{N}=9)$ \\
\hline Civic Organization Participation & 2 & 5 & 7 \\
\hline \multicolumn{4}{|l|}{ Age Range } \\
\hline $18-30$ & 0 & 1 & 1 \\
\hline $31-40$ & 0 & 0 & 0 \\
\hline $41-50$ & 2 & 0 & 2 \\
\hline $51-60$ & 0 & 3 & 3 \\
\hline $61-70$ & 0 & 3 & 3 \\
\hline \multicolumn{4}{|l|}{ Marital Status } \\
\hline Now Married & 2 & 4 & 6 \\
\hline Divorced & 0 & 1 & 1 \\
\hline Never Married & 0 & 2 & 2 \\
\hline \multicolumn{4}{|l|}{ Highest Education Completed } \\
\hline Some College or Less & 1 & 1 & 2 \\
\hline College Degree or Higher & 1 & 6 & 7 \\
\hline \multicolumn{4}{|l|}{ Reported Health Status } \\
\hline Excellent & 0 & 0 & 0 \\
\hline Very Good & 1 & 5 & 6 \\
\hline Good & 1 & 2 & 3 \\
\hline Fair & 0 & 0 & 0 \\
\hline Poor & 0 & 0 & 0 \\
\hline \multicolumn{4}{|l|}{ Household Income } \\
\hline$\$ 19,999$ or less & 0 & 2 & 2 \\
\hline$\$ 20,000-54,999$ & 1 & 4 & 5 \\
\hline$\$ 55,000$ or greater & 1 & 1 & 2 \\
\hline
\end{tabular}


Initial Interview

In the initial interview, participants were asked a series of questions based on quotes from prior focus groups conducted in the LMD. Data analysis of these interviews revealed a number of health concerns shared by participants. These concerns included the following: inaccessibility of healthy foods, sedentary lifestyles, unhealthy parental influence, and the need for nutrition education. Many of these concerns were summed up by a statement from one of the participants: Everything starts at home. Many participants expressed their belief that parents were the "gatekeepers" of health for their children. One concern unique to the Latina participants was acculturation. One participant said about the influence of acculturation, Latinos, when they come [to the United States], the majority of them eat healthy. You know, we snack on fruits and vegetables because that's the cheap thing in our countries. And then we come here, and we want to be Americanized, and we eat McDonald's. The health concerns identified from the initial interview are detailed in Table 3.

Table 3

Participants' Health Concerns from Initial Interview

\begin{tabular}{ll} 
Concern & Selected Quote(s) \\
\hline Inaccessibility of Healthy Foods & $\begin{array}{l}\text { "They [farmer's markets] are so expensive! Now, } \\
\text { speaking of the part of the community that we serve, } \\
\text { they can't afford to go to the farmer's market... This } \\
\text { place is so divided socioeconomically." }\end{array}$ \\
\hline "TV is okay. But it's not good for the children. \\
Especially spending their whole weekend, all day \\
$\begin{array}{l}\text { Saturday and Sunday laid up in the house watching } \\
\text { TV and buying all these video games. They even } \\
\text { bring them to church! They do, they don't ever stop } \\
\text { with them." }\end{array}$ \\
\hline
\end{tabular}


Table 3 (continued).

\begin{tabular}{|c|c|}
\hline Concern & Selected Quote(s) \\
\hline \multirow[t]{3}{*}{ Negative Parental Influence } & "Everything starts at home." \\
\hline & $\begin{array}{l}\text { "Parents really don't have time to observe their } \\
\text { children or take into consideration what they're } \\
\text { doing because they have their own things and the } \\
\text { children have their things." }\end{array}$ \\
\hline & $\begin{array}{l}\text { "The mothers that receive assistance, they don't buy } \\
\text { the food that's healthy..." }\end{array}$ \\
\hline \multirow[t]{3}{*}{ Need for Nutrition Education } & $\begin{array}{l}\text { "They have no idea what healthy is. You know, they } \\
\text { don't realize that six cans of Coke a day is } \\
\text { unhealthy. And you know, you realize that a can of } \\
\text { Coke is unhealthy, but they don't. So they need to be } \\
\text { informed." }\end{array}$ \\
\hline & $\begin{array}{l}\text { "I teach nutrition education myself. And I'm } \\
\text { learning that children don't even know how to spell } \\
\text { certain words; like in order for me to teach them } \\
\text { about the fruits and vegetables and the five food } \\
\text { groups, I have to teach them how to spell the food } \\
\text { groups before I can get into my lesson." }\end{array}$ \\
\hline & $\begin{array}{l}\text { "You go into the grocery sometimes and see two } \\
\text { baskets of food for one family, and they have } \\
\text { cookies, potato chips, candies, pops, and ice } \\
\text { cream... You know, the foods are high calorie foods } \\
\text { and no nutritional value, and we do need to be } \\
\text { redirected." }\end{array}$ \\
\hline Acculturation* & $\begin{array}{l}\text { "Latinos, when they come, the majority of them eat } \\
\text { healthy. You know, we snack on fruits and } \\
\text { vegetables because that's the cheap thing in our } \\
\text { countries. And then we come here, and we want to } \\
\text { be Americanized, and we eat McDonald's, you } \\
\text { know." }\end{array}$ \\
\hline
\end{tabular}

*Specific to Latina participants.

Participants also had positive things to say about their communities' health. One topic that was discussed in some capacity in every interview was gardening, including home gardening, gardening education in schools, and community gardens. When asked to 
describe what their ideal healthy community would look like, many participants stated that there would be gardens with produce available to everyone. One participant answered, A picture of everybody with a garden, eating healthy food they raised and sharing it with those who can't, so that we could bless and be blessed. Another participant explained that she had noticed many people returning to home gardening because of the expense of fresh produce at the grocery store; other participants shared this sentiment. Another positive theme that emerged from the initial interview was optimism. The participants expressed the need to stay positive about the health of the LMD. One participant said, We are already trying to teach them the danger of if we don't do something. But we want to show them some outcomes. I'm telling you all the bad things, but if I don't give you good things, I have nothing to rely on. Another said, We really are working hard. I can't knock us for that. Despite the challenges and concerns discussed, participants seemed hopeful that the LMD was on the verge of becoming a healthier region. As one participant described her ideal healthy community, It would look like an emerging balloon... I'm not satisfied with where I am, and I do know some steps to get better, so I'm on the way upward.

\section{Conceptual Framework}

From participants' photographs, journal reflections, and statements during the final interview, the researcher identified a number of prominent themes. These themes were used to develop a grounded theory-based conceptual framework. The core category, Hope for a Healthy Future, emphasized the participants' optimism about the current health of the LMD, as well as their hopes to create a healthier environment for their children. The Pillars of the Ideal Healthy Community were revealed through the 
photographs as well as the interviews. The five Pillars of the Ideal Healthy Community are Access to Healthy Food; Nutrition Education; Physical Activity; Safety and Cleanliness; and Role Models. Although each of these pillars had their own unique barriers and facilitators to implementation in the LMD, an overarching barrier (Resistance to Change) and an overarching facilitator (Spiritual, Familial, and Community Ties) that encompassed the overall health environment of the LMD were noted. The Latina participants also identified some barriers to health that were unique to the community of Latinos and Latinas living in the LMD. These barriers included dietary acculturation and being excluded from health promotion activities within the larger community. Facilitators included the closeness of the Latino culture and maintenance of cultural food traditions. However, these themes were not included as a part of the conceptual framework due to a small number of Latina participants. The conceptual model is illustrated in Figure 2. Each component of the conceptual framework is discussed in more detail below. 


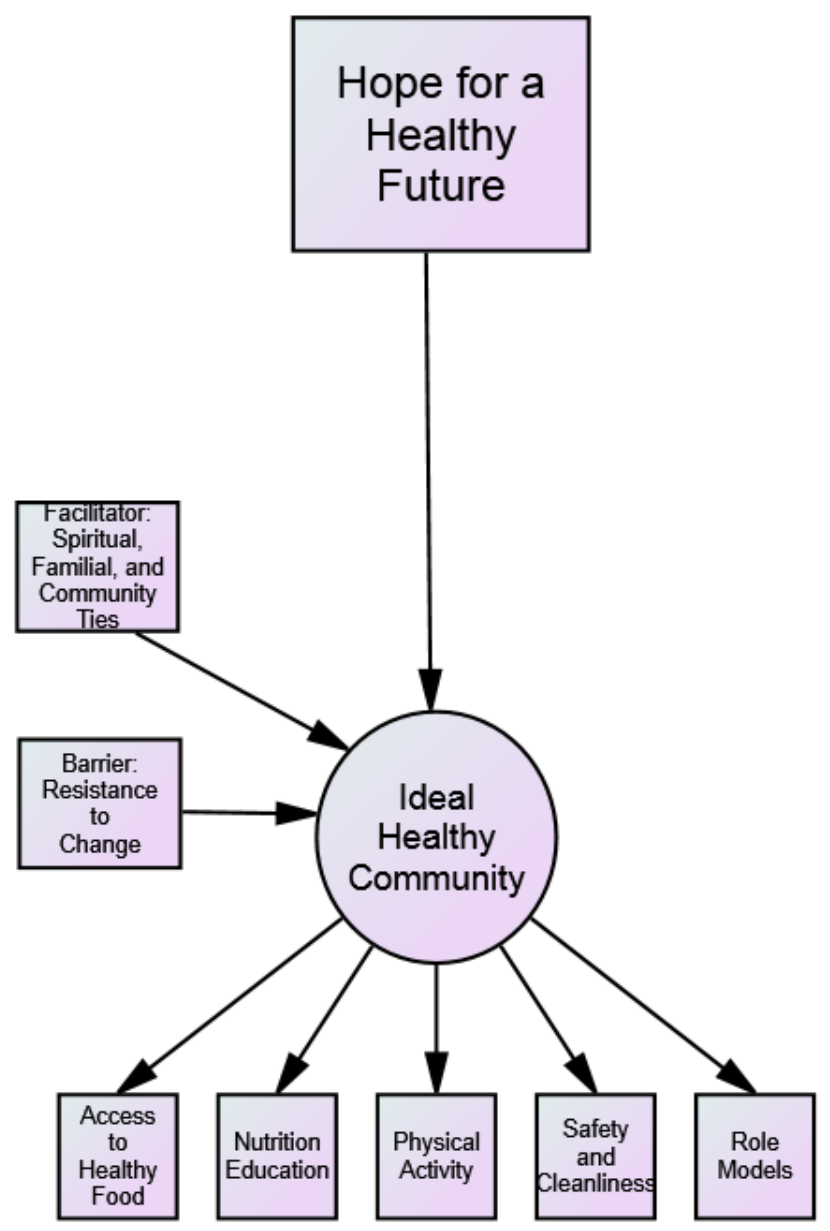

Figure 2. Conceptual framework: Health perceptions of minority women living in the LMD.

Hope for a Healthy Future

The participants' Hope for a Healthy Future was characterized by optimism about the future of the LMD. Many of the quotes were similar in nature to the optimistic statements the women made in the initial interview. One participant said, in reference to her community's health outreach programs, We really do a lot in our community; we have actually made great things. The participant who described her ideal healthy community as an "emerging balloon" in the initial interview said in the final interview, The balloon is rising, all we have to do is hop in it...before it goes all the way up! 
Many participants expressed that they wanted the LMD to be a healthier region so that the children in the region had the opportunity to lead healthy lives. Figure 3 is a photograph that one of the participants took of some her young relatives playing together at a family reunion, illustrating this core category. In her journal reflection about this photograph she wrote, When I look at this picture, I think about the life these little girls must live out. With good health and nutrition, these little girls will grow up to be healthy women.

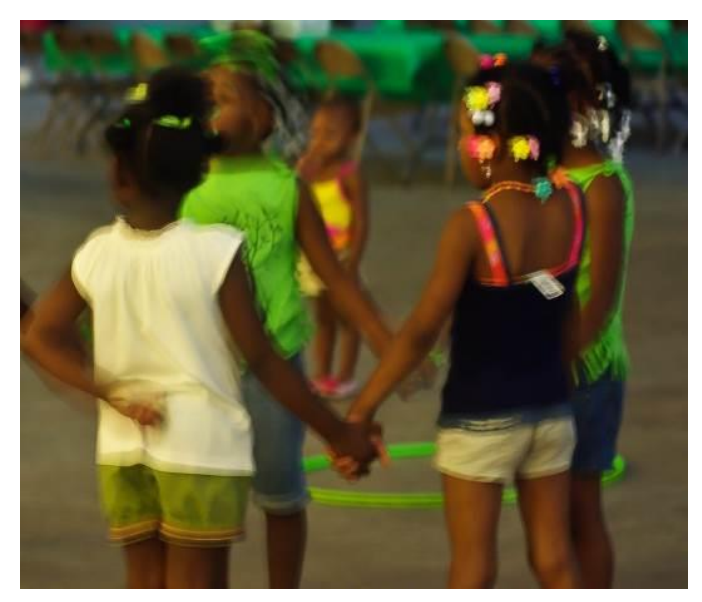

Figure 3. Participants were concerned about creating a healthy future for children. Pillars of the Ideal Healthy Community

Access to healthy food. Access to Healthy Food was identified as one of the most important aspects of a healthy community by participants, and a number of facilitators and barriers to healthy food access in their communities were shared. The women were very enthusiastic about home gardening as a way to access healthy food, save money, and prepare for the future. One participant observed that many people were returning to home gardening in response to rising food prices and limited options at the grocery store: They are getting back into it [gardening], because it's becoming so expensive, and you only have one grocery store. Another said, It's [farming] an activity for you to do, and then 
it's income and it supplies your daily routine: eating [laughs], which is a must. Figure 4 is a photograph of a cow owned by one of the participants captioned in her journal as, These are pictures of farm-locally raised beef along with the clean grass and water they are fed; and preparations of food for the farms are done at the local slaughterhouse. You grow your own food; therefore, you know what's in it. One Latina participant pointed out that many Latinas keep small gardens at their homes for similar reasons: to save money and eat healthier. In addition to home gardening, the women also supported farmer's markets and community gardening efforts.

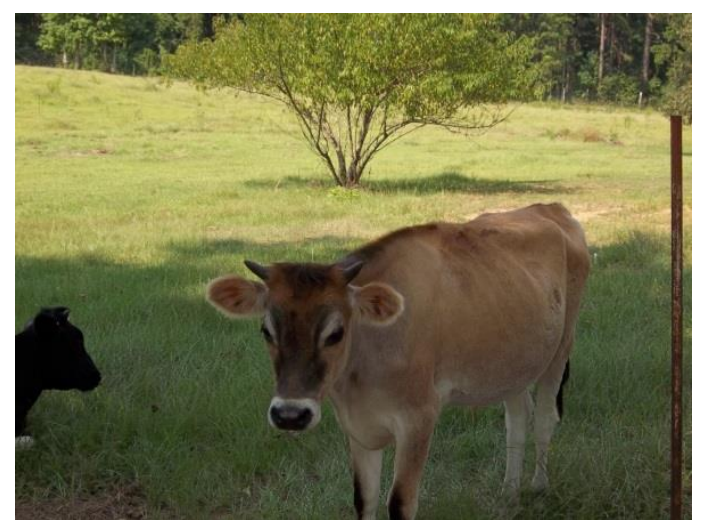

Figure 4. Participants were particularly interested in home gardening, community gardening and farmer's markets.

A few of the women were involved in community gardens, and the others were very receptive to the idea. The existing community gardens that participants were involved in were conducted through schools. One participant was involved in a gardening and nutrition education program for elementary school children, as well as a "reading fruit tree orchard." She explained, "The idea is to get a reading fruit tree orchard, where the kids can come outside and sit under the trees and read and just enjoy the fruit. And, the fruit will be free to the public!' Figure 5 shows the participant and students planting trees at the orchard. 


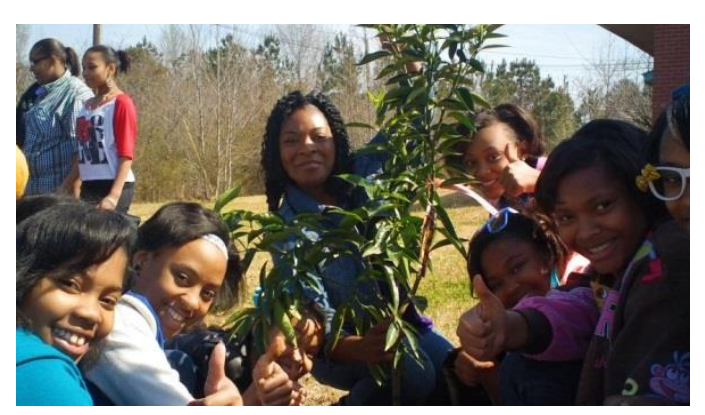

Figure 5. Some of the participants were involved in community gardening programs through schools.

Participants were also enthusiastic about farmer's markets and eating locallysourced food. Figure 6 is a photograph of locally-grown watermelon. This photograph represents the importance of Access to Healthy Food to the participants by highlighting the many ways that local produce benefits the community. The participant who took the photograph explained, I just wanted to express that there are some local farmers and that in addition to purchasing produce that's good for our health, that we will also be helping our local economy. Another participant said, The farmer's markets help us to keep our revenue within the city and then, also, it supplies good, fresh fruit and vegetables to the people, which is good nutrition for our community. So therefore we are supplying a need and we are also making a profit off of our own, you know.

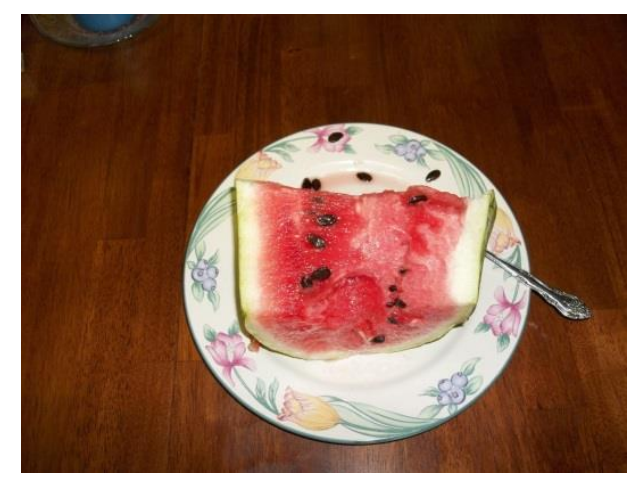

Figure 6. Locally grown foods were important to participants because they were healthy and they kept money in the LMD's economy. 
The participants also cited the availability of healthy options in restaurants as a facilitator to health in the LMD. One participant explained that she loved a particular fast food restaurant because they provided hot, healthy meals that included baked fish and steamed vegetables. She said, Even if you don't cook, if you have a super super crazy schedule and you don't have time to cook, you have to go out to eat, you can still eat healthy. She also added, A lot of the fast food restaurants are changing the way they season their foods, and they're changing the oil that they cook in because of the health food craze. So they're trying to help us out, or at least pretending they are. Another participant said, You can still go out and eat. A lot of the time my husband and I, we seldom cook, we go out and eat. When you go, you gotta know what to get. However, one of the Latina women stated that she felt there were no healthy options at restaurants in her community. In addition to this perceived barrier, there were many other reported barriers to healthy food access within the community as well.

One of the most significant barriers to healthy food access reported by the women centered around negative influences in the home and family. Of primary concern was the observation that most families no longer cook. When asked why obesity was such a problem in the LMD, one participant answered, People don't like to cook. Another participant explained, Our schedule just run so hectic now. People just don't take the time to cook. I even find myself falling prey to that 'cause sometimes you come home, you just too tired to cook. Lack of cooking leads many individuals and families to a dependence on convenience foods and fast foods. Figure 7 is a photograph of a typical meal of one of the participant's relatives. She explained, Lots of mayonnaise and cheese curls. Said he was hungry, he didn't have time to cook, so that's what he ate. 


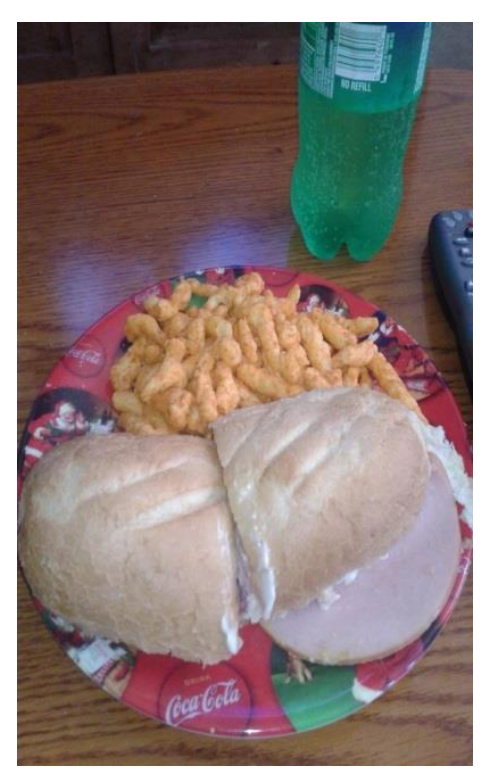

Figure 7. Participants perceived that many people in the LMD ate unhealthy foods because they were more convenient than healthy foods.

Other participants were disturbed by some of the choices made by low-income families at the grocery store. Many participants shared that they frequently observed some of the younger, low-income families stocking up on junk food at the store. One participant said, A lot of people that are on public assistance, I can see them in the grocery store sometimes with a basket just filled to the top with mostly junk food. Another said, You don't like to cook, you getting a lot of food stamps, you got a lot of children... you don't buy what you need, you buy what's easy. The participants felt that this "new generation" of parents were setting children up for failure. One said, I remember years ago, parents were different. The parents were concerned about their children, just like we were, but today we got a whole new generation and they seem to put themselves first. But, we want them to put God first, their children next. This perceived difference between the younger and older generations arose during the analysis as a significant barrier to eating healthy food. 
Generational differences were cited as a significant barrier to healthy food access among both Black women and Latinas. One Latina participant expressed, The girls, American food and the older ones, Mexican food. Another explained, You'll see a bowl of fruit in every home of the Latinos. Now, instead of a bowl of fruit, the younger ones have cornflakes as snacks... You know, it's cheaper and easier. Figure 8 is a photograph of one of the Latina participant's daughters with three bags of chips. She wrote about the photograph, My 'American' daughter would eat this for breakfast if I let her.

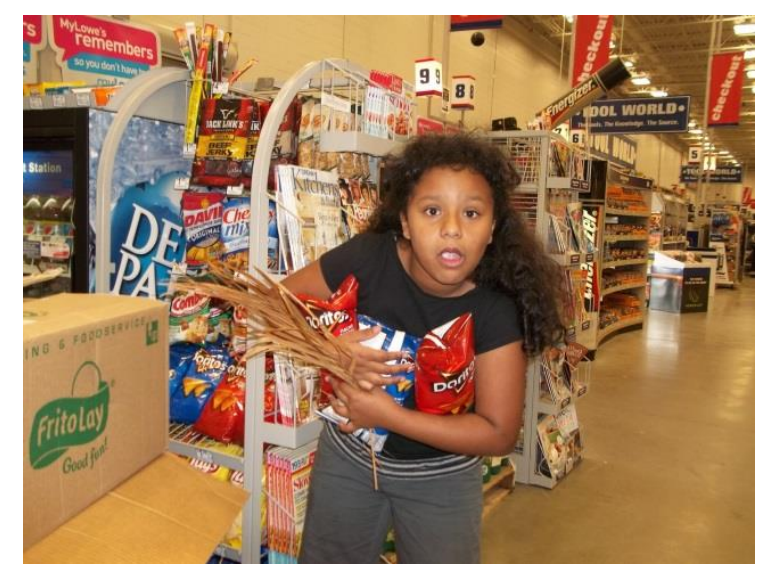

Figure 8. Participants noticed a definite difference in the food preferences of the older generation and the younger generation.

At a family reunion, one of the Black participants observed, Most of the younger people went to the sweet stuff first. Older people drank the water. The Black participants also expressed that gardening was an "older folks" activity. One said, When I was comin" up, it seemed like everybody in my neighborhood had a garden... as they [older people] die out, the gardens gonna die out! Other Black participants expressed similar sentiments. Overall, barriers to healthy food access and consumption echoed a theme that arose in both the initial interview and the final interview: "Health starts at home." Ideally, nutrition education will help LMD residents to improve their access to and consumption of healthy foods. 
Nutrition education. Black women and Latinas expressed a desperate need for nutrition education in the LMD. Many participants perceived that health disparities in the LMD stemmed from a lack of nutrition education. One woman said, I think education is just the foundation. A lot of people do what they do because that's all they know. Of significant concern to many participants was a belief that LMD residents did not believe certain unhealthy foods were actually unhealthy. Figure 9 is a photograph of a menu board at a popular fried fish restaurant in the LMD. The participant wrote, As you can see, there are lots of menu items that may not seem healthy, but to Deltans and southerners here in the South, who may think it's healthy. When asked why people think this food is healthy, she answered, Because that's what's being served in restaurants every day.

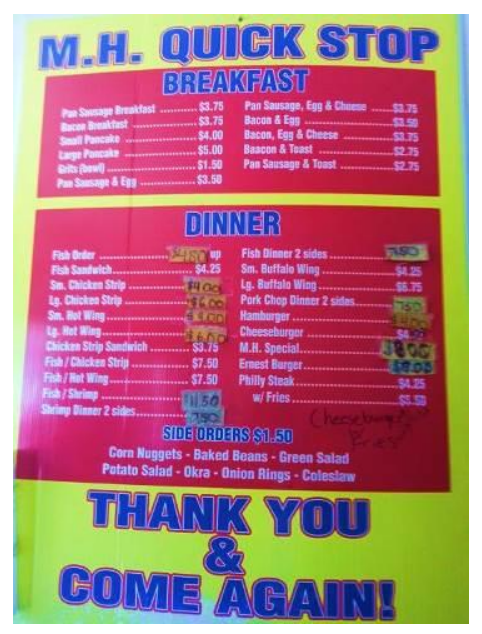

Figure 9. Participants shared how easy it was to get fried food in the LMD and also that many people probably did not realize that fried foods were unhealthy.

Similarly, participants shared concerns about some LMD residents' lack of awareness concerning soda. One participant wrote in her journal under a photograph of a poster about sugary drinks, Everyone was shocked when they learned how much sugar was in drinks of different kinds. The two Latina participants explained that many Latinos 
replaced "waters"- a traditional beverage containing watered-down fruit juice and a small amount of added sugar — with sodas when they immigrated to the United States. One said, When they do come here all of that [waters] is replaced with soda. So they'll drink like five or six sodas a day, no problem - because they don't realize.

The participants had many different ideas about how nutrition education could reach the public, some of which were already in place. One participant said, Education is always good. But sometimes you just have to carry it to them.... I know that's bad, but it's a fact. You just have to carry it to the people's house. Other participants participated in nutrition education outreach activities through food pantries, civic and social organizations, or their jobs in schools and in public health. One woman who was involved in a local food pantry said, We put handouts in their bags [at the food pantry] to tell them how to cook their foods and then parts of eating healthy, nutritious foods. A participant who works in a high school created the poster shown in Figure 10. She explained that it was to promote nutrition and remind them [the students] to eat the rainbow.

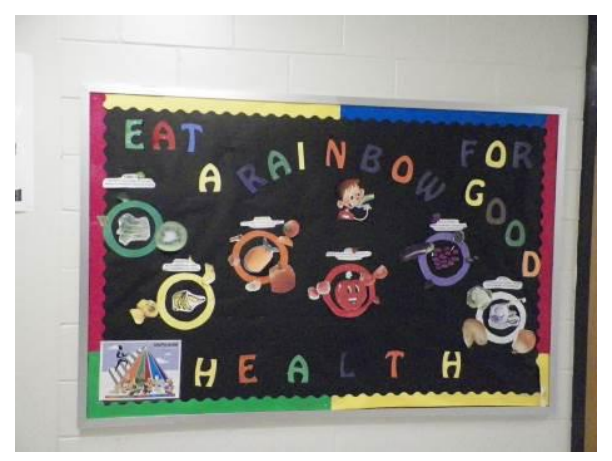

Figure 10. Many participants were involved in nutrition education activities through schools, churches, or community organizations.

Physical activity. Many participants expressed the importance of physical activity to health, especially for children. This emphasis on physical activity allowed it to stand 
alone as its own Pillar of the Ideal Healthy Community. Overall, the women seemed to agree that their communities provided a fair number of resources and opportunities for residents to engage in physical activity. The participants took many photographs of playgrounds, parks, and community centers. Figure 11 is a photograph of a playground inside of an apartment complex. One participant wrote, All the modern apartment complexes in my community have playgrounds, providing youth a healthy alternative to watching television and playing video games.

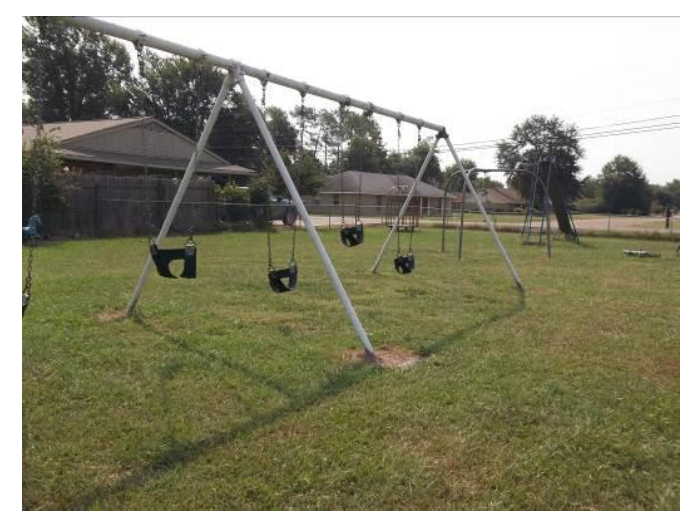

Figure 11. There were many recreational facilities, like playgrounds and parks, in the LMD.

However, despite the availability of recreational facilities, there were still a number of barriers to physical activity in the commuity. One of the most significant problems related to lack of physical activity was leading a sedentary lifestyle. One participant said of people who watch television all day, They don't try to find anything else to occupy their time. They'll sit there and they're satisfied, and you should never be satisfied with where you are... if you have met your limitations, you must be God. Another felt that "couch potatoes" were bringing down the health of her entire community: If we can get those couch potatoes up and moving, even just a little bit, that would affect our whole health - the whole big picture as far as health goes in our 
community. A trend in the photographs revealed that there was many parks, playgrounds, gyms and recreation centers but there were no people present. When asked about this apparent underutilization of facilities, one participant answered, They are somewhat underutilized, based on our population, and We can be taking care of ourselves much better if we just would use it. The final major barrier to physical activity in the LMD was a lack of safety and cleanliness, which also represents one of the pillars of the Ideal Healthy Community.

Safety and cleanliness. This pillar was one that first arose in the participants' photographs, and it was also unique to Black participants. However, subjects related to Safety and Cleanliness were some of the most frequently photographed. Participants were very outspoken about the role of safety and cleanliness in community-wide good health practices. When asked why safety and cleanliness were so important to health, one participant said, Cleanliness is very important to your health and safety and the environment. Another said, Safety is taking measure to prevent accidents, and accidents can really be a health hazards. Some of the health hazards the participants photographed included potholes, abandoned homes and cars, broken playground equipment, and litter. Participants photographed clean, well-kept playgrounds, homes, and public spaces as examples of safety and cleanliness in an ideal healthy community. Although it can be difficult to rectify the participants' concerns about lack of safety with the availability of seemingly clean and safe facilities, some of these inconsistencies may be the result of regional differences among the participants. Figure 12 is a photograph of a clean, well-lit walking area in a downtown area in the LMD. 


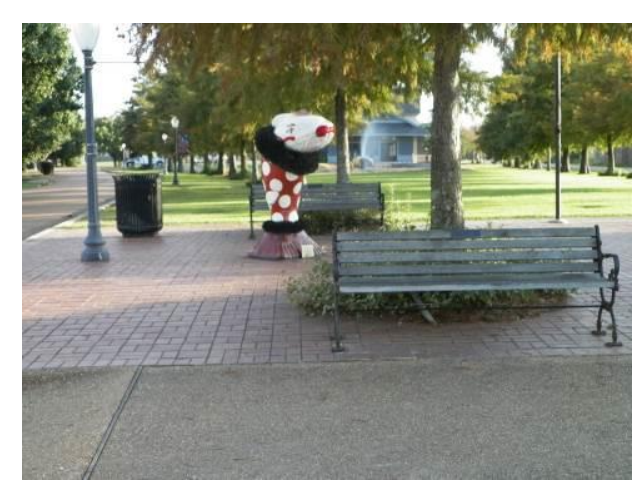

Figure 12. Participants took many photographs of good examples of safety and cleanliness in their communities.

Participants expressed a definite need for public spaces that were safe and clean, as many participants took photographs of unsafe and unclean areas that were problems in their communities, particularly areas where children gathered or played. Abandoned buildings were a major concern. Participants expressed their concerns that abandoned homes were a tempting play area for young children. One woman said, Children could just be in there playing, and then someone could... go in there. A negative person, an adult could go in there and damage. So it's not good. Another participant worried that these abandoned buildings attracted vagrants. She said, I just think that's not a safe environment with drug dealers or either crackheads being able to get up in abandoned buildings. Four participants photographed abandoned buildings and homes like the one in Figure 13, which the participant captioned Another abandoned business, broken window great for drug traffickers and homeless persons. 


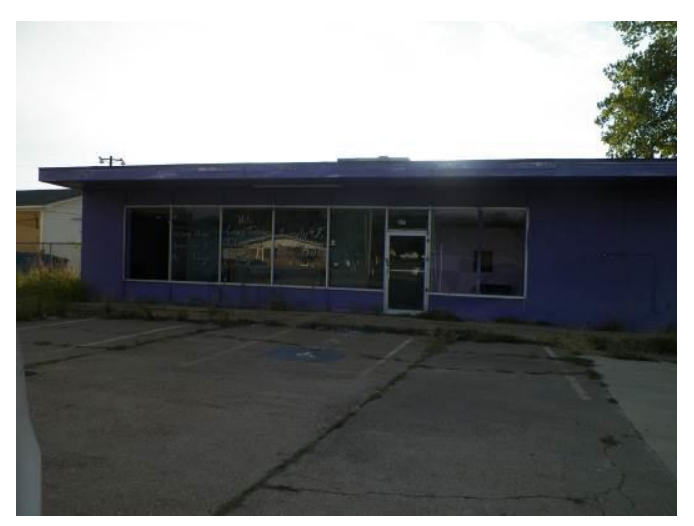

Figure 13. Participants took many photographs of abandoned homes, buildings, and cars. They perceived these abandoned facilities as dangerous to all community members, but especially to children.

Designated play areas for children were also problematic, with litter, broken equipment, and unkempt grass being some of the most frequently cited problems. Figure 14 depicts a set of bleachers on a children's playing field that has no seats.

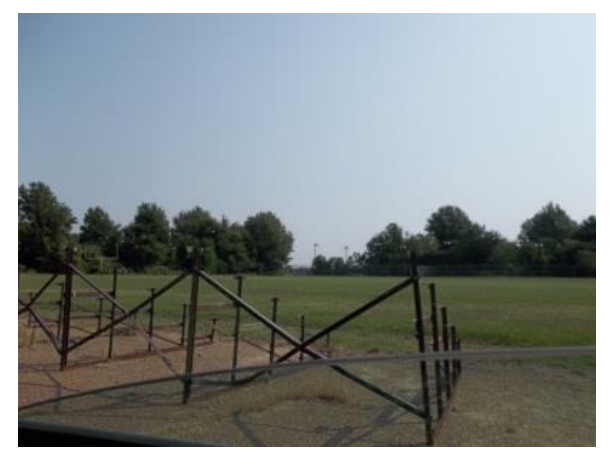

Figure 14. Unkempt grass, broken equipment, and litter were some of the most photographed subjects related to safety and cleanliness.

As one participant stated, It's [a play area] not being taken care of the way it's supposed to be as a community place where children play. Cleanliness is very important to your health and safety and the environment. Black women in the LMD perceived Safety and Cleanliness as vitally important to the health of their communities.

Role models. The final pillar of the Ideal Healthy Community that the participants identified was Role Models. Most participants were actively involved in nutrition and 
public health outreach in some capacity and expressed an earnest desire to set positive, healthy examples for communities. Participants also expressed the importance of having healthy role models for the children in the communities. One participant said that she enjoyed being a role model to children: I went to one school during lunch time and just by me going by and giving them high fives. I'm like 'oooh, you ate your vegetables, give me a high five!' That started a whole row of kids to eat their salad. A Latina participant who worked in public health shared, We need to set the example. We need to exercise more, that'll help with the stress and all of this dependency on caffeine.

In addition to sharing a desire to be role models, the participants shared stories and photographs of role models in their families and communities. One participant shared that the pastor at her church was a very influential role model in her community. She said, He plays a vital role in motivating people, so I think now he's trying to start a health ministry... and church is very, very persuading. Like, if you get the preacher behind you on something, he can just tell everybody else and they'll just come. Figure 15 is a photograph of this participant's great aunt, who is a matriarch and a role model in her family. Her journal reflection stated, At 89 years old, my great aunt is still hanging in there... I feel that because of a life of some kind of good health practices, she made it to 89 years of living. 


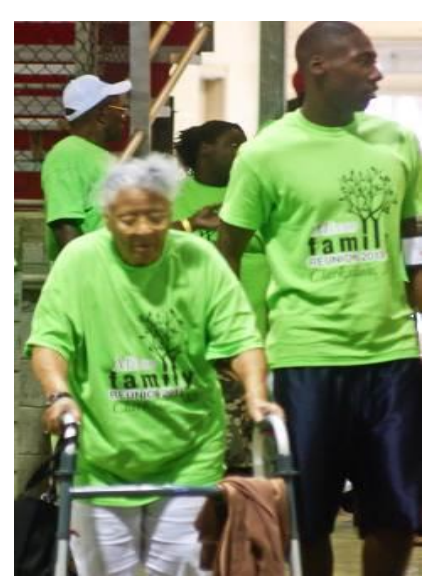

Figure 15. Participants wanted to lead healthy lives so that they could be role models to future generations, like this woman.

Another participant shared photographs of her son and her niece, explaining how her son was now a role model for the entire family after losing a significant amount of weight. Figure 16 is a photograph of her son and her great niece. She wrote in her journal, A healthy young man who has lost 60 pounds within the past two years and is still eating healthy... his niece trying to follow in his footsteps.

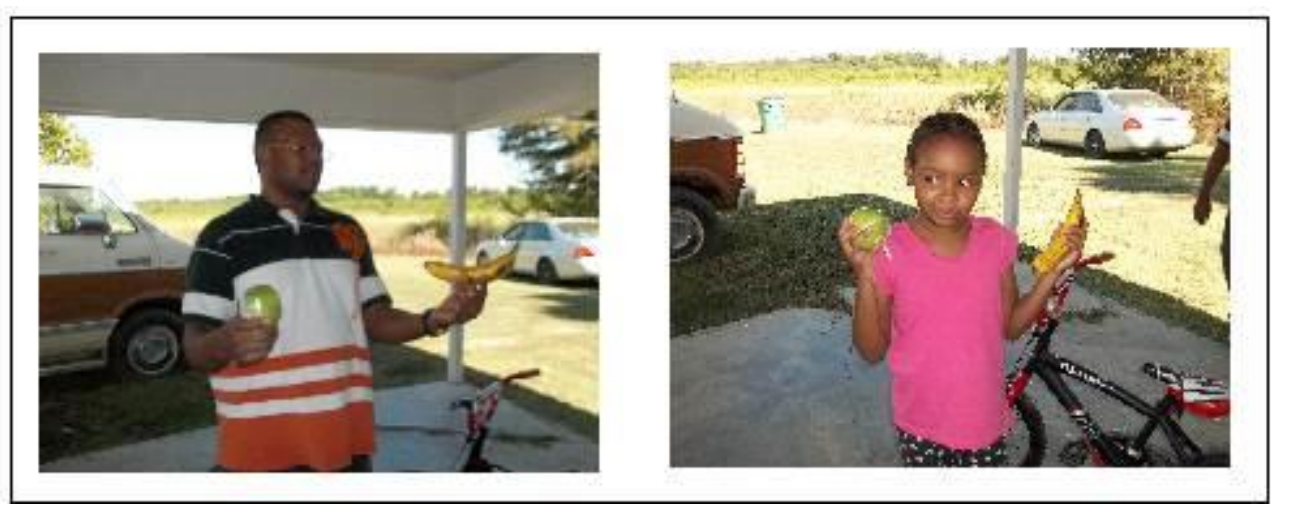

Figure 16. These photographs, of a man who lost 60 pounds and his niece, highlight the importance of healthy role models in the LMD.

The Health Environment in the LMD

During data analysis, one barrier (Resistance to Change) and one facilitator (Spiritual, Familial, and Community Ties) to health in the LMD seemed to impact most or all of the pillars of the Ideal Healthy Community. When combined, these represent 
major facets of the participants' perceived health environment within their communities. Two environmental forces seemingly in conflict with one another were Resistance to Change serving as an obstacle to improving health in the LMD and Spiritual, Familial, and Community Ties serving as motivation for improving health in the LMD.

Resistance to change. Participants perceived the LMD region, as a whole, as resistant to change, especially change that involved outside influences. One participant said, We have a system that fights improvement. They [community leaders] don't want anybody else coming in telling them what to do. One of the Latina participants shared her struggle as a Latina married to a Black man to be fully accepted by anyone living in the LMD. He's a black American, she said, and that's also a big no-no for the Latino community. There is [a lot of racial division in the LMD]. I mean the people that I love are white, people that I love are black and Hispanic, and then over here it's almost like you're asked to choose, you know. And I don't wanna choose. She went on to add, about the LMD as a whole, They've all grown with each other and it's hard to get into those little groups that they've made themselves. And it's with just about every race and ethnic group here in the Delta. It's a little more difficult in the Delta than it would be elsewhere. Racial division was not the only area in which LMD residents were resistant to change, however. Another participant found it very difficult to motivate people to get involved in their communities. She said, Getting somebody to do it [community service], you have to wait on their time. Especially if they're doing it for free... and so a lot of times people are motivated by money... If there is no money behind it, there's not many true commitments. Although participants were highly involved in health promotion and very enthusiastic about the future, they realized that many of the people living in the LMD were not as 
excited about health as they were. One participant said, We are so unhealthy here in the Delta, and you know... Sometimes it hurts my feelings to look at people, how they're just killing themselves with the things that they eat. And then, people in general, when you try to help them, they get offended and hurt, and they think that you're trying to impose your views on them. So, I've learned now to be quiet... the culture is so anti-change. So antichange. They think that if they change, it's a bad thing. They think if they speak up, it's something bad.

Spiritual, familial, and community ties. Spiritual, Familial, and Community Ties emerged as a very significant theme during data analysis. Spirituality, family, and community seemed to serve as powerful support networks for participants. Spirituality was of particular importance to the Black participants. One of the participants explicitly said that her work in health promotion was a higher calling and that it was her responsibility to serve and better her community. I think we should serve God by serving each other, she said. I know we put here to be our brother's keeper. Figure 17 is a photograph of a family matriarch leading prayer. The participant's journal reflection of the photograph was, I truly feel that you can't have health and wellness without prayer! Having a spiritual relationship with the heavenly Father or having some form of religion is essential in wellness! 


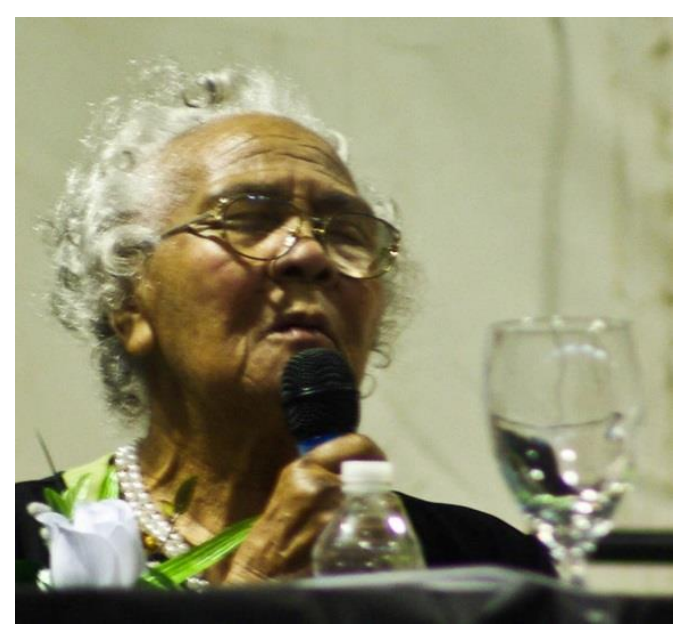

Figure 17. Prayer and spirituality were very important to the Black participants.

Bonds between family members were also a noteworthy facilitator to health in

the LMD. Families were a source of encouragement, happiness, and emotional and social support. Figure 18 is a photograph of a large dinner gathering at a family reunion that took place in the LMD. In her journal, the participant who took this photograph wrote, Family fellowship and dinner is an essential part of Deltans. Many African-Americans fellowship by eating together on various occasions. Food choices aren't always the healthiest, but like the cliché states, 'food for the soul.' Because the consumption of food with family is a time to laugh, cry, and build relationships. Mississippians love family dinner time. 


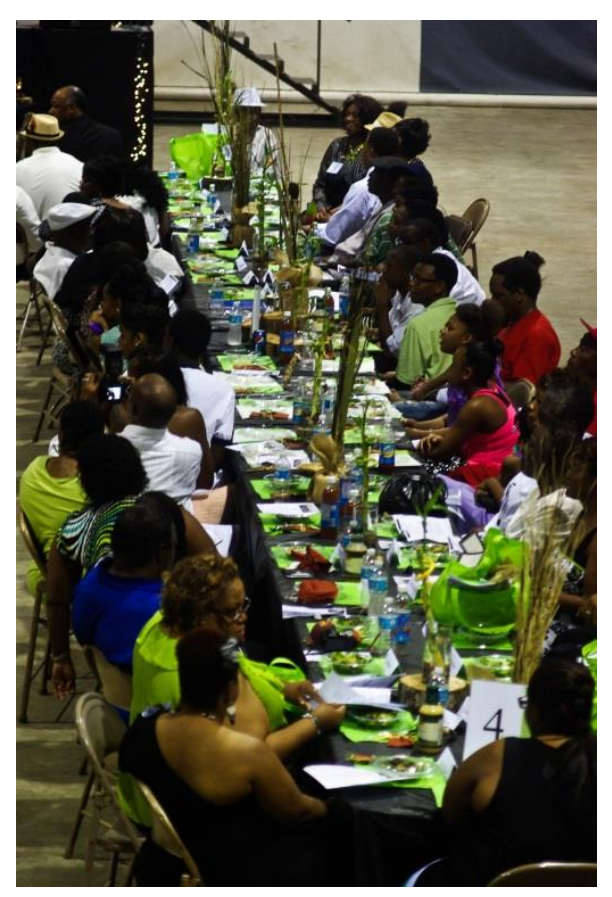

Figure 18. Many photographs illustrated the importance of family.

Family members also served as "enforcers" of healthy diet changes and provided emotional and social support. Like my uncle, he had a triple bypass surgery and he had the salt shaker, trying to shake the salt on all the food and we was like 'stop, ' one participant said. I think family can stop certain things, they can prevent certain deaths, 'cause you know you don't need to be eating all this salt, we just snatch it away from him.

Figure 19 is a photograph of a Sunday dinner with one of the participant's families.

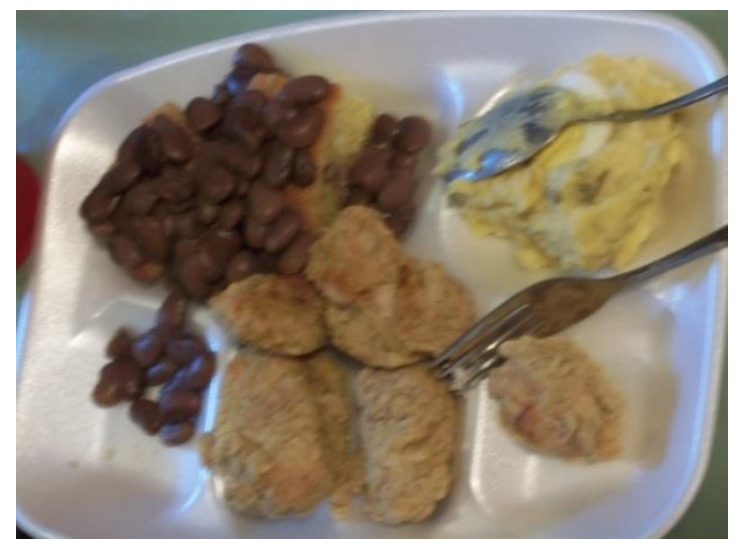

Figure 19. Family members were a source of encouragement, and they also enforced healthy behaviors when necessary. 
This participant explained that she and her siblings ate dinner together every Sunday, and they always tried to accommodate each other's' special diet and nutrition needs. Those [chicken strips] are baked because my brother that had high blood pressure earlier than we did, the participant said. This past Sunday was mine [turn to cook], and I tried to watch the sodium, you know. The concept of home as the starting point for learned habits and behaviors — both healthy and unhealthy — was evident in photographs and in the final interviews, as it was in the initial interview. Family ties do have the potential to serve as a barrier to the general population of the LMD, such as the negative parental influences that were repeatedly discussed by participants. However, family ties were a facilitator to health for participants in this study. One participant said, A healthy lifestyle begins at home. It depends on how well you eat nutritious and healthy foods, exercise, and communicate with each other. It is a journey.

Participants also shared the ways in which bonds between community members were important facilitators to health in the LMD. Most of the participants were actively involved in health promotion in the LMD and felt personally responsible for helping to improve health in the region. Discussions were threaded with participants' social involvement in churches, schools, and community organizations. Figure 20 is a photograph of a participant's church. She writes, Assembled in the House of the Lord, I've come to realization and discovery that when I attend church services regularly, it seems that my weeks go smoother. My well-being seems to be in a more relaxed place and my focus is sharpened. Like many southerners, church is a place of worship and can play a huge part it health and wellness for the soul. 


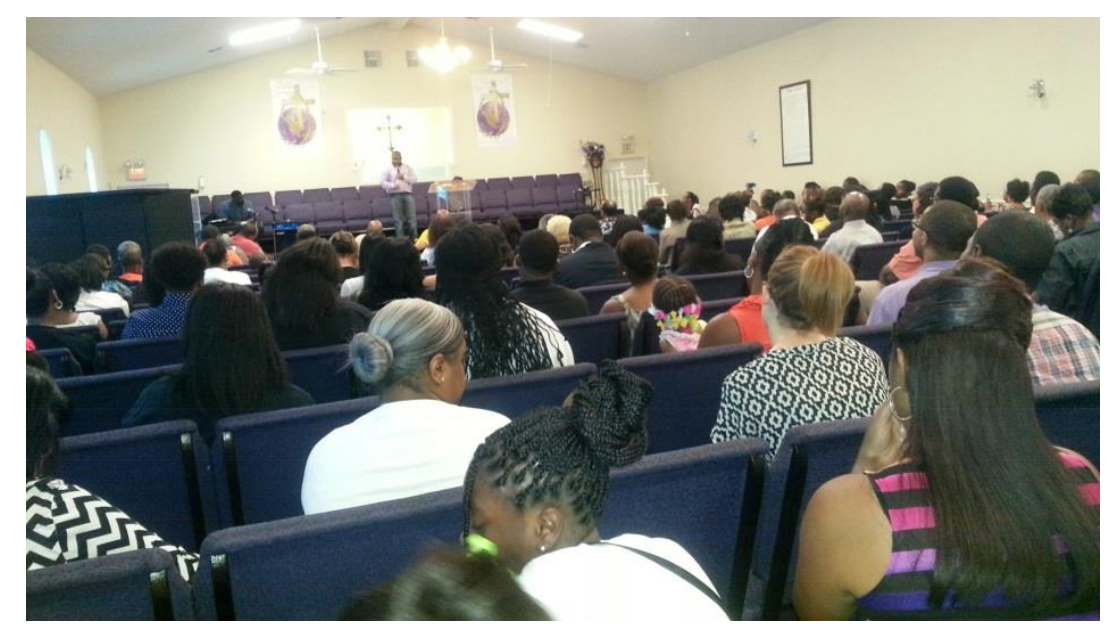

Figure 20. Churches were the cornerstones of LMD communities, according to participants.

Another participant observed, Several churches in our area... have started a health ministry... I think those are the kinds of things we do need to start doing on a regular basis. Many participants, including the two Latinas, agreed that churches would be very effective at diffusing nutrition education to the general public in the LMD. School personnel also seemed to be very involved in health promotion. A few of the participants were teachers, and they provided numerous examples of nutrition education and physical activity programs that took place at schools. In addition to students, activities organized through the school attracted adults. Figure 21 is a photograph of a high school football stadium on game night. A participant who worked at this school explained that the school had recently hosted a "meet the team" night, where students and their parents could walk laps on the track with the football team. All this happened right before the first game, she said, and we had the parents to walk, you know, everybody walked. So it was a community-wide walk around the field, for like a mile. 


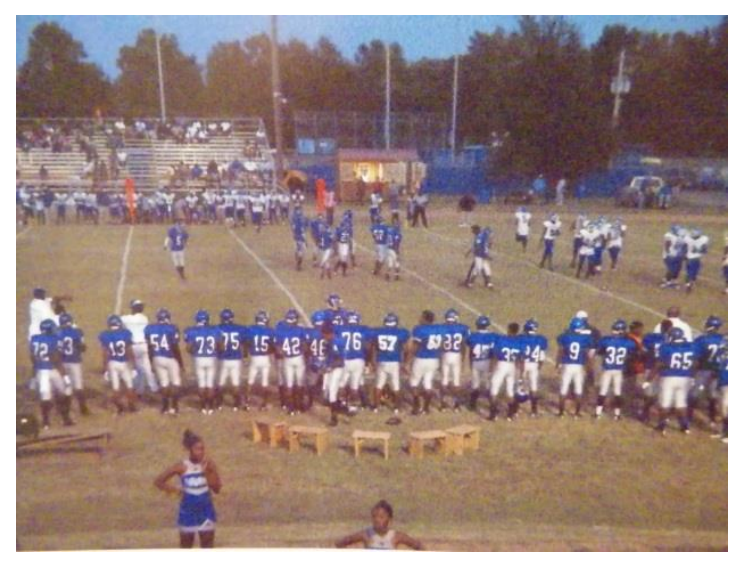

Figure 21. School functions allowed opportunities for both children and adults to engage in physical activity.

Participants were involved in many different social and civic organizations. Some worked in public health and at schools, while others volunteered in non-profit organizations or worked with public health researchers in the LMD. According to one participant, many different societies and organizations hosted "walk for a cause" events. Some people will show up just for a cause and don't realize that your own body is a cause, she said. That's one thing that I think we could do is capitalize on the kinds of activities that we already have and stress the importance of letting it be a continuous thing. Figure 22 features photographs of some of the different health-promoting organizations and activities in the LMD. The consensus among the participants seemed to be that, although there were plenty of organizations and activities working to promote health, it was still difficult to reach people and get them invested in their health and the health of their families. 


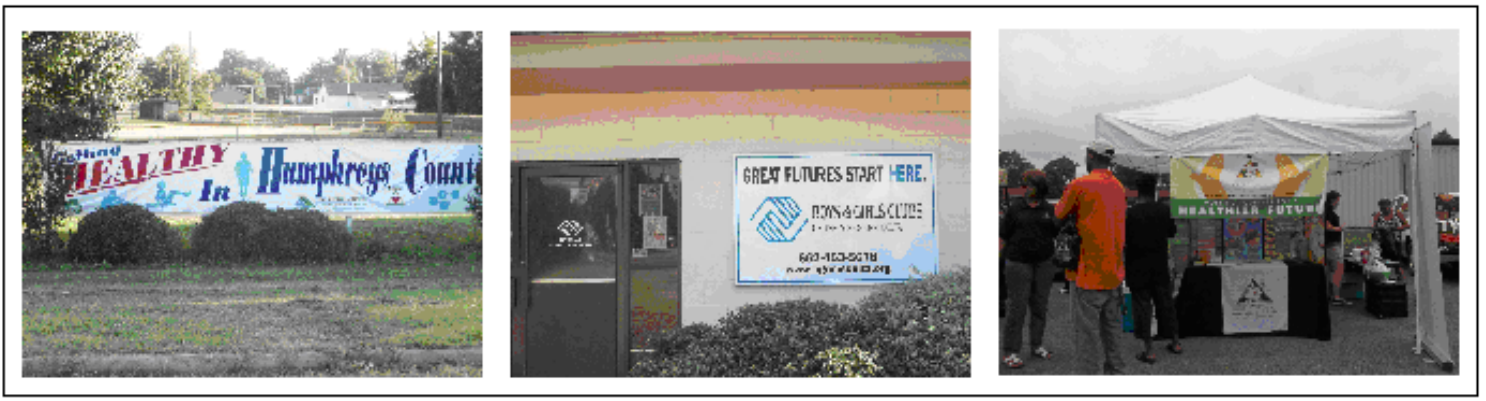

Figure 22. Participants took many photographs of health promotion organizations and activities.

\section{Latinas in the LMD}

Although in many instances the Latinas and the Black women perceived health similarly and identified similar facilitators and barriers to health, some barriers and facilitators to health unique to the Latina population did arise from the photographs, journal reflections, and interviews with the Latina participants.

Exclusion from the larger community. A significant barrier to health in the community of Latinos and Latinas living in the LMD was exclusion from health promotion activities in the larger community. This sense of being excluded was revealed in both the initial and final interviews with the Latinas. One participant, speaking through a translator, said, We don't know, and if we don't have someone to teach us, we'll never know. She wishes that there were more programs where the [Latino] community could learn how unhealthy they are eating. We need to learn, we need to teach, we need more programs to teach us. Another participant explained that, despite the large number of health promotion activities and programs in the LMD, Latinos were still excluded. This was due in part to the language barrier between Latinos and others in the LMD. Latinos were not made aware of these programs through advertising or outreach. They have so 
many programs where they can teach us, she said, but they're not available in our language, and they [Latinos] don't even know that it's there.

Dietary acculturation. Acculturation primarily affected health through changing dietary habits, according to the Latina participants. Adopting some of the healthier components of an American diet was viewed as a positive healthy behavior by the Latina participants. One said, I've learned a lot of new things here too, with the canola and the bulgur... so I incorporate it into my rice dishes, you know the arroz con pollo. I'll put some canola in the arroz con pollo, which is the traditional rice dish that most Latinos eat. So I'll put that in my food, and instead of white rice we'll do brown rice. Figure 23 is a photograph of cereal and milk at a Latina participant's home. About this photograph, she wrote, This is my breakfast. $2 \%$ milk. I am trying to buy soy milk, but it costs more money and it is important to invest in ourselves if it will improve our health.

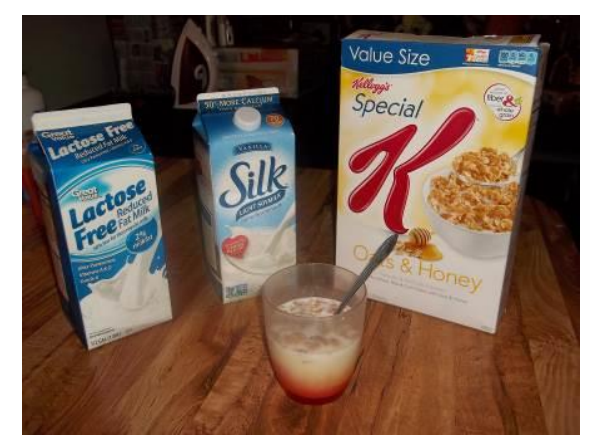

Figure 23. In some instances, adopting American foods helped the Latina participants to be healthier.

However, dietary acculturation seemed to be far more damaging to health than it was promoting to health. One issue that appeared very significant to the two Latina participants was the problem of traditional Latino foods being prepared "Americanstyle," with processed ingredients, added fats, or added sugars. One participant's daughter worked at a Mexican restaurant in the LMD. Figure 24 is a photograph of a 
typical meal prepared by her at the restaurant, made with white rice and a good deal of fat. The participant wrote, Green enchiladas, rice, and salad. It can be nutritious if we change the way we cook. For example brown rice cooked with olive or vegetable oil.

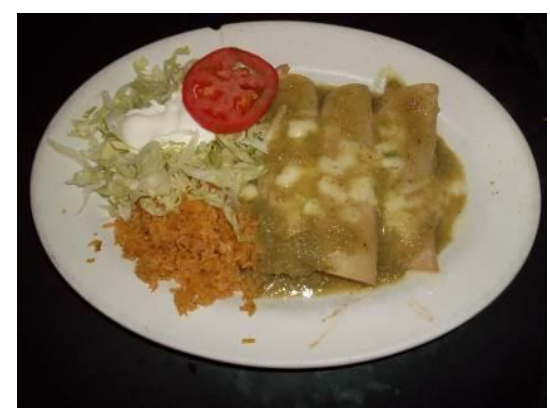

Figure 24. Mexican restaurants often prepared their foods "American-style," making them very unhealthy.

Another Latina participant said, The tacos [at the Mexican restaurant] are made with a lot of fat. They put a lot of fat, even on the tortillas. When asked to explain further and to talk more about why even traditional Latino foods are made unhealthy, she explained that most Latinos in the LMD used processed, convenience items instead of making their foods at home. This problem of dietary acculturation also extended to the adoption of unhealthy American foods.

Dietary acculturation as a barrier to health for the Latino population in the LMD is closely aligned with a barrier identified by the Black participants: generational differences. Similar to the observations of the Black participants, Latina participants shared that younger Latinos appear to prefer processed convenience foods and other unhealthy foods to traditional meals. As one participant explained, In the morning they'll [Latinos in the LMD] drink a soda with their breakfast. At lunch, it's two or three servings of soda, and then the tortillas, they just keep coming... The children, even the younger ones. Easy access to fast food in America also served as a detriment to health, 
according to the Latino participants. Over here [in the United States], it's cheap [fast food]. That's a cheap thing to eat, you can get something for a dollar. Over there [in Mexico], we can't afford to eat at a fast food. Overall, the participants expressed that dietary acculturation was more harmful to the health of Latinos than beneficial.

Simpatia. Another aspect of Latino culture that was both a barrier and a facilitator to health was simpatia, which describes the importance of close, interpersonal relationships to Latinos as a group. As a barrier to health, these closely-knit relationships contributed to the change-resistant, "closed-off" community of Latinos living in the LMD. As one Latina participant said, You have to get the trust of the Latino community. Even as a Latina herself, she experienced a great deal of resistance in attempting to engage the Latino community in health promotion activities in the LMD. I don't speak like them. Also my accent is different... it's difficult to get in, she said.

As a facilitator to health, these close relationships encouraged friends and family members to exercise together and motivate one another to practice good health habits. Figure 25 is a photograph of a group of friends walking in the park. The participant who took the photograph explained that she and her friends met often to walk, and occasionally they brought their husbands with them. Friends are important, she said, For example, they encourage me to walk over there at the park. 


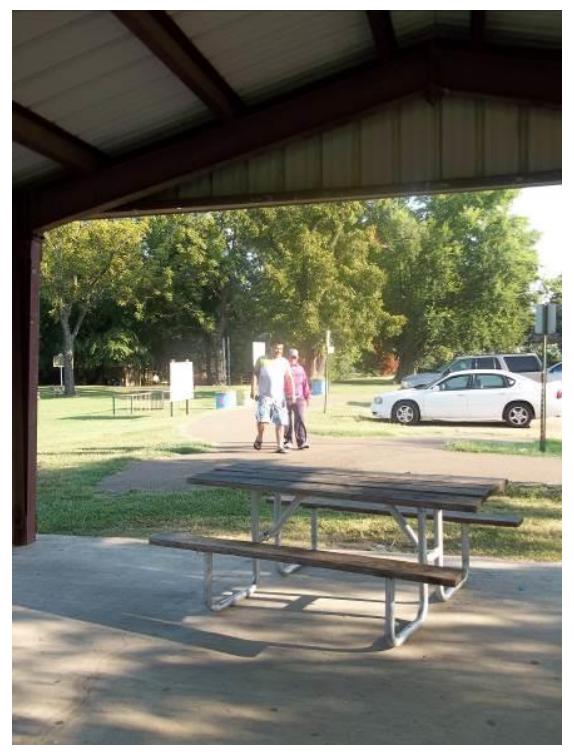

Figure 25. Friendships were important to the Latina participants, and these friends encouraged each other to engage in healthy behaviors.

Family members also served as role models in this community according to the Latina participants. The Latina participants, like the Black participants, expressed a sincere desire to be seen as positive healthy role models for their families. One said, When they go out to eat, you'll have my seven year old asking for Indian food as opposed to McDonalds. They'll ask 'well, I wanna eat Thai today' because that's what we do at home. Figure 26 is a photograph of one of the Latina participant's daughters. She wrote, Waffles is a good food to give them a taste from time to time and then have them exercise in the yard 30 minutes to an hour. The Latina participants used their relationships with friends and leadership roles within their families to improve their individual health and the health of their loved ones. 


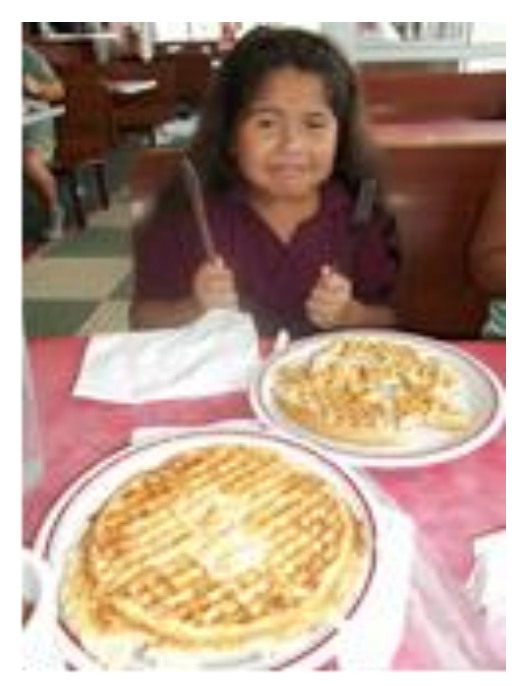

Figure 26. A Latina participant instilled healthy habits in her children by having them exercise after eating a big meal.

Maintenance of cultural food traditions. A major facilitator to health that was unique to the Latina participants was the maintenance of cultural food traditions. The Latina women spoke extensively about the ingredients and preparation techniques used in traditional Latino cooking and expressed a desire to retain these traditions for the sake of health. They both agreed that the traditional diets from their home countries, Ecuador and Mexico, were much healthier than a typical American diet. Figure 27 is a photograph of the Ecuadorian participant's mother. The participant wrote about her, My mom has the healthiest diet of any person I know. Eats organic food, whole grains, has a green smoothie daily. Has never been sick. Exercises daily. Never fries food, no cans, no boxes. Everything made from scratch. She is from Ecuador. In the second interview, the participant said that her mother already ate in a very controlled manner coming from our country. 


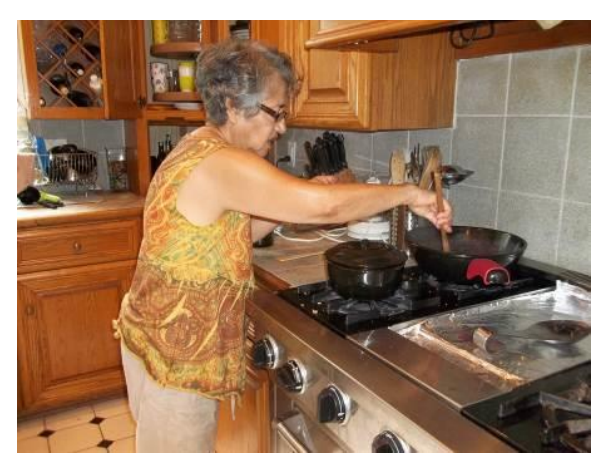

Figure 27. Cooking and eating traditional foods was perceived by the Latina participants as much healthier than American foods.

The participants explained that, although tortillas and rice were an important part of their traditional diets, they were prepared with very little oil—unlike the tortillas and rice served at Mexican restaurants in the United States. Where I'm from, one participant said, the tortilla does not get prepared with oil. They may get a little drop of oil, and over here they slather it with oil. Figure 28 is a photograph of a healthy, traditionally-prepared meal that one of the Latina participants cooked.

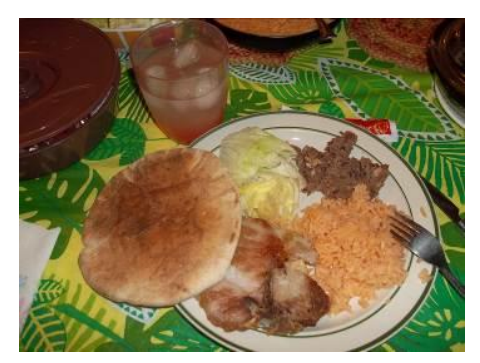

Figure 28. The Latina participants tried to maintain their cultural food traditions by cooking Mexican and Ecuadorian foods at home.

One participant also explained that a typical American breakfast containing eggs, meat, and toast was reserved for special occasions in her home country, and even then the portions were much smaller. That's a big breakfast that we eat daily here, she explained, It's a treat for us [in Ecuador]. Figure 29 is a photograph of a typical Latino breakfast of café con leche y galletas - coffee with milk and crackers. 


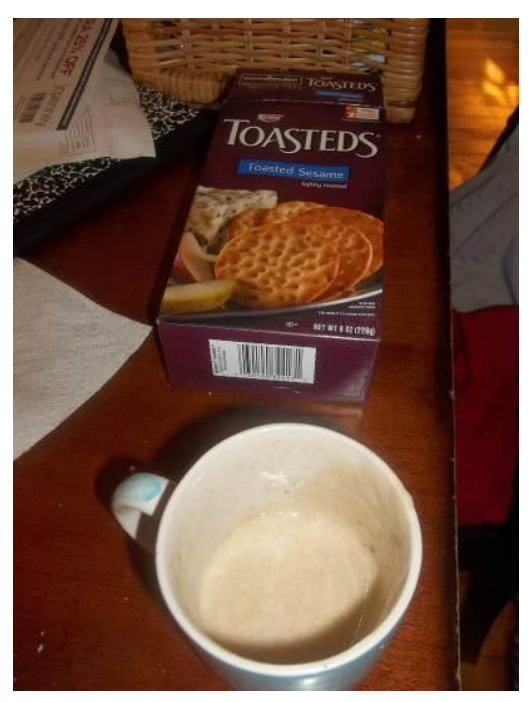

Figure 29. A typical Latino breakfast of coffee and crackers is much smaller than a standard American breakfast containing eggs and toast.

In addition to the themes that were brought out in the conceptual framework, the Latina participants' health perceptions were shaped by other facets of their experiences as well. Exclusion from health promotion activities within the larger community, dietary acculturation, simpatia, and maintenance of cultural traditions shaped the health experiences and perceptions of these two Latina women living in the LMD. 


\section{CHAPTER V \\ DISCUSSION}

Research on the health perceptions of minority women living in rural areas, and the LMD specifically, is limited. However, many of the findings from this project aligned with findings from existing research (Beydoun \& Wang, 2008; Carver, Timperio, \& Crawford, 2008; Connell et al., 2007; Haskell, Lee, Pate, Powell, \& Blair, 2007; Perry et al., 1988). Although the Pillars of the Ideal Healthy Community are a new framework, the individual pillars have all been identified as important to improving community health in prior research (Beydoun \& Wang, 2008; Carver et al., 2008; Connell et al., 2007; Haskell et al., 2007; Perry et al., 1988). The core category, Hope for a Healthy Future, also aligned with existing research on health perceptions among minorities (Maton \& Wells, 1995; Williams, Hewison, Stewart, Liles, \& Wildman, 2012).

Resistance to Change and Spiritual, Familial, and Community Ties - the overarching barrier and facilitator, respectively_ have also been previously identified as having a profound impact on the daily lives of residents of the LMD. Finally, the findings about the community of Latinos living in the LMD also aligned with existing research on Latino immigration, dietary acculturation, and simpatia. The findings of this study have allowed the health perceptions of minority women living in the LMD to be organized into a conceptual framework, with the Pillars of the Ideal Healthy Community helping to illustrate the participants' Hope for a Healthy Future.

In a qualitative study on the health perceptions of African fathers living in England, researchers asked participants about their perceptions related to fatherhood, health, and healthcare (Williams et al., 2012). The researchers found that participants 
were optimistic about the health of their children, and they were trying their hardest to be good fathers and to instill healthy habits in their children. This attitude of optimism is similar to the core category identified in this study, Hope for a Healthy Future. Hope for a Healthy Future was characterized by both optimism about participants' individual health and fervent desire to improve the environment of the LMD to make it a healthier place for participants' children and grandchildren. Researchers have also found that optimism, particularly optimism that is rooted in religious faith, is associated with better mental and physical health (Maton \& Wells, 1995). The optimism among participants fueled their desire to help the LMD become more like the Ideal Healthy Community.

The five pillars of the Ideal Healthy Community have been discussed and studied extensively in prior research, although as separate facets of health and not merged into a single framework (Beydoun \& Wang, 2008; Carver et al., 2008; Connell et al., 2007; Haskell et al., 2007; Perry et al., 1988). The first, Access to Healthy Food, has been researched in the LMD. Researchers have found that many LMD residents have limited access to healthy food due to price and decreased availability in rural areas (Connell et al., 2007; Farley et al., 2009). The participants also indicated that many people in their communities ate unhealthy foods due to convenience. Researchers have found that this sentiment, that unhealthy food is more convenient, is common among many people; in one study, researchers found that people who rated convenience as the primary factor influencing their food choices tended to have unhealthier diets (Pollard, Steptoe, \& Wardle, 1998). However, the participants felt that community gardening, home gardening, and farmer's markets could drastically improve the LMD's access to healthy food. In a study on fruit and vegetable intake among participants in an urban area, 
researchers found that those who participated in a community garden consumed more fruits and vegetables than those who did not (Alaimo, Packnett, Miles, \& Kruger, 2008). Many of the participants in the present study also observed that low-income families receiving food assistance purchased large amounts of unhealthy foods. Bhargava and Amialchuk (2007) found that increased consumption of added sugars among families receiving food assistance "displaced" vital nutrients in the diet such as protein, fiber, calcium, and iron. Poor diet quality has also been associated with a lack of nutritionrelated knowledge (Beydoun \& Wang, 2008).

Nutrition Education was discussed at length by the participants, and therefore was identified as one of the Pillars of the Ideal Healthy Community. While nutrition education alone is not enough to improve diet quality, it is one factor that has been identified as a contributor to increasing HEI scores (Beydoun \& Wang, 2008). The participants' suggestions to use community organizations, churches, and school in order to diffuse nutrition education to the public also aligned closely with current research on the importance of social support in minority communities; researchers have found that Black individuals who were involved in strong social support networks such as churches, community groups, and families reported higher overall wellbeing than those who were not involved in these social support networks (Taylor, Chatters, Hardison, \& Riley, 2001).

Physical Activity was also frequently cited as an important component of health by participants. The American College of Sports Medicine and the American Heart Association recommend that adults between the ages of 18 and 65 get at least 30 minutes of moderate-intensity exercise five days per week. This level of exercise has been shown 
to help improve cardiovascular and mental health, increase muscle mass, and promote energy balance (Haskell et al., 2007). Physical activity can also decrease the risk of many chronic diseases, including diabetes and cancer (Warburton, Nicol, \& Bredin, 2006). These clear benefits to physical activity had been observed and experienced by many of the participants, which warranted the inclusion of Physical Activity as one of the pillars of the Ideal Healthy Community.

Safety and Cleanliness, the fourth pillar, has been linked to improved health and increased physical activity (Zoellner, Hill, Zynda, Sample, \& Yadrick, 2012). This theme of Safety and Cleanliness is one that did not arise until the participants began to take photographs, but it quickly became of the most prominent themes in the entire study. Of specific importance to the participants was the availability of safe and clean public spaces for children to play in. In other PhotoVoice projects assessing the health perceptions of low-income minority populations, safety was also a prominent issue (Findholt et al., 2010; Hannay et al., 2013; Torres et al., 2013). Many participants in these projects photographed broken playground equipment, unsafe sidewalks, and other examples of unsafe areas where children congregated within their communities. In a review of existing literature conducted by Carver and colleagues (2008), the researchers found a clear association between low levels of physical activity and a lack of safety within communities. In this way, the two pillars of Physical Activity and Safety and Cleanliness were closely intertwined.

The final pillar, Role Models, was one that seemed to resonate deeply with the participants. The participants expressed, through words and photographs, an earnest desire to be positive, healthy role models for their communities and their families. There 
is relatively little research available on the influence of role models specifically on nutrition and health outcomes. However, the role of parents as role models for their children has been researched extensively. Many researchers have concluded that parents play a profound role in shaping the health behaviors of their children, and that parental influence can affect a child's future health outcomes (Perry et al., 1988; Lindsay, Sussner, Kim, \& Gortmaker, 2006). This research parallels the women's consensus that "health starts at home." Church leaders have also been studied for their role modeling potential (Lumpkins, Greiner, Daley, Mabachi, \& Neuhaus, 2013). The study participants considered church leaders key stakeholders in their communities. In existing research, Black church leaders have been identified as key stakeholders in their communities due to the high rates of church attendance and religious participation among Blacks (Ellison, 1995; Lumpkins et al., 2013). The concept of Role Models as a pillar of the Ideal Healthy Community is also closely related, especially in existing research, to the primary environmental facilitator that was identified in this study: Spiritual, Familial, and Community Ties.

Religion and spirituality are very important to Black communities, particularly in the southeastern United States (Ellison, 1995; Ross \& Wheeler, 1971). Many Black participants in this project cited their Christian faith as a motivation to volunteer in health promotion activities. In other studies, religiosity and church involvement have been linked to better mental health, stronger social support networks, and higher self-esteem for Black individuals (Ellison, 1995; Taylor et al., 2001). In a grounded theory study on Black clergy and their ideas for health promotion within the church, researchers found that these clergy felt health promotion was part of their God-ordained mission (Lumpkins 
et al., 2013). Clergy also felt their influence could be used to encourage the community to engage in healthy behaviors.

Family also emerged from the study as a very significant source of support. Prior research into the cultural constructs of familismo, personalismo, and simpatia-umbrella terms for the close, interpersonal connections between Latino families and friends--in Latino communities reveals the importance of family and close social bonds in this ethnic group (Galanti, 2003; Triandis et al., 1984). Family closeness is also important to Blacks. In one study on subjective well-being, family closeness, and religiosity in Black Americans, researchers found that living in the South and having close relationships with family were highly correlated with participants' overall satisfaction with life (Taylor et al., 2001). Church, family, and community are all closely intertwined within the Black communities in the LMD. For this reason, churches are often the target of nutrition and public health interventions. Despite the positive aspects of these strong support networks for Blacks and Latinos in the LMD, community-wide Resistance to Change was still a significant barrier to achieving the Ideal Healthy Community.

Resistance to Change was cited by many participants as a large problem in the LMD. This Resistance to Change did not manifest as a barrier to any specific pillar, but instead as an overarching barrier that affected every aspect of health promotion in the LMD. In particular, the participants brought out the underlying racial divisions, distrust of outside authority figures, and lack of involvement in community activities as the most detrimental aspects of this Resistance to Change. Although it is a challenge to find published research assessing the scope of this Resistance to Change, the history and current climate of the LMD are telling of the region's struggles to "keep up" with the rest 
of the United States (Fontenot et al., 2010; Rodriguez, 2012). As previously discussed, the LMD has a long history of Black enslavement on plantation farms (Fontenot et al., 2010). Since the Civil War era, Blacks have struggled as an oppressed minority in the LMD. This oppression is visible through the high poverty and unemployment rates, low educational attainment, and health disparities among Blacks in the LMD (Fontenot et al., 2010; LMDNIRC, 2010; McCabe-Sellers et al., 2007; Mirvis et al., 2004; MSDH, 2012a; Wang \& Beydoun, 2007). Latinos struggle similarly, as many come to the LMD as poor migrant workers and deal with poverty and health disparities at nearly equal levels (Rodriguez, 2012). Latinos immigrants also struggle with the detrimental effects of acculturation on their diet quality and the subsequent health effects, which is reflected in the Latina participants' photographs and reflections (Lara, Gamboa, Kahramanian, Morales, \& Bautista, 2005).

In addition, the LMD also has been declining economically in recent decades (Gray, 1991). Although it was once a booming agricultural center, technological advances have allowed most farms to operate with significantly fewer employees (Fontenot et al., 2010). This environment is not friendly to economic growth, which has resulted in an economically “stagnant” LMD (Gray, 1991; Lentz \& Oden, 2001). Reconciling the Resistance to Change that is present in the LMD with the strong interpersonal support networks within LMD communities poses a significant challenge to public health researchers hoping to decrease health disparities in the area.

\section{Strengths and Limitations}

There were several strengths to using PhotoVoice methodology and grounded theory in this study. PhotoVoice and grounded theory are uniquely suited to one another 
due to their similar aims (Lopez et al., 2005). The main goal of PhotoVoice projects is to positively influence public policy, while the main goal of grounded theory is to form a conceptual framework based on study findings, which can then be used as a tool to improve interventions and outcomes in similar communities (Corbin \& Strauss, 1990; Wang \& Burris, 1997). The use of photographs and journal entries prompted the participants to think deeply about the health of their communities and elicited thoughtful conversations about their health perceptions. The conceptual framework that resulted from this study describes the relationship between participants' hope for the future and their ideas of what defines the Ideal Healthy Community. The framework can be used as a tool for future researchers to key in on the aspects of health that are important to community leaders in the LMD. The researcher introduced a high level of rigor by completing a full transcription of each interview and by having the results reviewed by colleagues who were knowledgeable about the nutrition and cultural aspects of the LMD. However, despite the strengths of this study, there were some limitations.

A lack of combined PhotoVoice and grounded theory literature could introduce methodological weakness because of a lack of consensus regarding the most proper way to conduct these types of studies. An additional weakness unique to PhotoVoice methodology is the large volume of data collected. From each participant, approximately 30 photographs, 30 journal entries, and two transcripts were analyzed. This surplus of qualitative data can cause issues with organization and analysis (Nykiforuk, Vallianatos, \& Nieuwendyk, 2011). Qualitative data analysis can also be fairly subjective (Madill, Jordan, \& Shirley, 2000); however, the researcher tried to minimize this bias by fielding the input of colleagues who were experienced with nutrition and public health 
interventions in the LMD. An additional limitation of the study was that only two Latinas completed the study. This limitation brings into question the reliability of comparing their perceptions to those of the Black participants $(n=7)$. For this reason, health perceptions that were unique to the Latina participants were excluded from the conceptual framework. A final limitation of this study is that it assessed the health perceptions of women who were already actively involved in health promotion activities in the LMD. Although this limitation may have resulted in findings that do not accurately represent the health perceptions of minority women in the LMD as a whole, it does allow an opening for future researchers to continue studying the health perceptions of minority populations in the LMD.

\section{Future Research}

There are many different directions in which future research on the health perceptions of minorities in the LMD could be taken. As mentioned above, this study focused only on women who were already actively involved in health promotion activities in the LMD. In order to get a more accurate picture of the health perceptions of minorities in the LMD as a whole, studies could be conducted with men and with individuals who are not community leaders. The LMD is a fairly large geographic area, consisting of 133 counties in Mississippi, Louisiana, and Arkansas. The participants in this study were all from the state of Mississippi, so future research could be done to identify regional differences in health perceptions among LMD minorities. However, the study of health perceptions is done in vain unless findings are used to strengthen and refine future health interventions. Ideally, findings from this project will be used as a 
starting point for future researchers to develop nutrition and public health interventions targeted at health disparate individuals in the LMD.

\section{Conclusion}

The LMD, with its high minority population and prevalence of low SES as well as health disparate individuals, is frequently the target of nutrition and public health interventions designed to help decrease the burden of health disparities. In order to design effective interventions, researchers must have a firm understanding of health perceptions held by residents of the LMD. In this study, a combination of PhotoVoice methodology and grounded theory was used to illuminate the health perceptions of Black women and Latinas living in the LMD. These women were actively involved in health promotion activities, and many of them could be considered community leaders. Photographs, journal entries, and quotations reflected a relentless optimism—participants' Hope for a Healthy Future for themselves, their families, and their communities. This hope fueled a desire to work towards the Ideal Healthy Community, which consisted of five pillars: Access to Healthy Food, Nutrition Education, Physical Activity, Safety and Cleanliness, and Role Models. Resistance to Change was identified as a significant barrier to health that affected the whole process, while the significant facilitators to health were identified as Spiritual, Familial, and Community Ties. Because of these unique barriers and facilitators to health in the LMD, positive change will likely need to come from within the LMD - from people who care deeply about the health of the region, such as the participants in this study. This "model" of the health perceptions of minority women living in the LMD was illustrated in the grounded theory-based conceptual framework that arose from this study. Ideally, the findings of this study will be used to facilitate 
positive policy changes such as improving maintenance of public spaces; increasing funding for community gardens, farmer's markets, and health initiatives; and developing community nutrition education programs. Likewise, findings could help researchers design more effective health interventions for minorities in the LMD. 


\section{APPENDIX A}

\section{INFORMED CONSENT FORM}

\section{The University of Southern Mississippi Authorization to Participate in Women's Perceptions of The Health of The Delta Using Visual Depiction and Journal Reflections Research Project}

The purpose of this research is to explore health perceptions among women belonging to social and civic organizations using visual depiction (PhotoVoice) and journal reflections in the Lower Mississippi Delta.

Participants must be at least eighteen years of age to participate.

The minimum requirements for you as a participant include:

- Attending an initial interview to discuss prior research related to this project, training on the project procedures, and informing of your responsibilities. The interview is expected to last one to one an half hours.

- Taking pictures with a provided digital camera of participant's community related to healthy lifestyle activities and record notes about the photos taken in a provided journal. The PhotoVoice process may take up to 2 weeks to take up to 30 pictures. If you take any photos of people, including yourself, you will need to obtain a Photo Release Consent Form provided by the researcher.

- When you have completed the photo assignment, you will mail the memory card in the camera to the researcher in the postage-paid envelope provided or upload the photos to a computer and email them to the researcher.

- Attending a second interview to discuss photos and journal reflections. The interview is expected to last one to one an half hours.

All interviews will be recorded using a digital voice recorder and a note taker will be present so as not to miss any pertinent information discussed. At any time, you may request that the recorder be turned off. You also have the right not to answer any information you choose. Each recording will be transcribed into a written form (transcript). Recordings and transcripts will be kept in a locked file cabinet, and your identity (if you choose not to be identified by name) will not be disclosed. In order to maintain confidentiality, no personally identifiable information such as name or phone number will be asked or associated with photos or journal reflections. Only the researcher will have access to the files collected. All data files will be destroyed at the end of the study. The results of this project will be used in a presentation for the Committee on Services and Resources for Women at The University of Southern Mississippi and may be presented in published articles.

Benefits associated with this project may include the valuable insights you may gain about the health of your community. You may also enjoy the creative components of this project, giving you the opportunity to express yourself both visually and in the written form. Additionally, you will be allowed to keep the camera (up to a $\$ 50$ value) and a $\$ 50$ 
gift card will be issued at the end of the second interview to compensate for your participation and time during this project.

Risks associated with this research project may include safety when taking pictures as well as the safety of the photo subjects. You will be instructed on safety and respect as described in the PhotoVoice Guidelines that you will receive during the training at the initial interview. Other inconveniences to you may be the time it will take to take the photos, write in the journal, mail the camera and journal to the researcher, and attend the interviews.

Participation in the project is completely voluntary, and you may withdraw at any time without penalty, prejudice or loss of benefits.

Questions concerning the research, at any time during or after the project, should be directed to Holly Huye at 601-266-6023 or at Holly.Huye@usm.edu. Final results may also be obtained by request by phone or at this email address at the completion of the study.

This project and this consent form have been reviewed by the Human Subjects Protection Review Committee, which ensures that research projects involving human subjects follow federal regulations. Any questions or concerns about rights as a research participant should be directed to the Chair of the Institutional Review Board, The University of Southern Mississippi, 118 College Drive \#5147, Hattiesburg, MS 394060001, (601) 266-6820.

I have been given a copy of this consent form and have been given an opportunity to ask questions. I am satisfied with the answers that have been provided. With my signature, I give my consent to participate in the research study.

Print Name of Participant

Signature of Participant

Date

Signature of Researcher

Date 


\section{APPENDIX B \\ PARTICIPANT INFORMATION PACKET}

\section{Getting Started: PhotoVoice Project Steps}

\section{Step 1. Take pictures. Think about these guiding questions:}

- What activities best show health or a healthy lifestyle in my community?

- What activities reflect the opposite of a healthy lifestyle in my community?

- What in my life or community has helped me live a healthy lifestyle?

- What in my life or community has prevented me for living a healthy lifestyle?

- What do I want to tell other people about living a healthy lifestyle?

- What are my hopes for the future? And what might help me get there?

Step 2. Write down the date, time and place of the photo you took and a description of the picture. You can write any additional reflections you may have about the photo.

Step 3. When you feel you have taken enough pictures that represent the health of your community (about 30 pictures), you may transfer the photos to your computer using the instructions in this packet and email them to the project leader (holly.huye@usm.edu) or mail the memory card (inside the camera) with the journal in the self-addressed, pre-paid postage envelope provided. Whichever method you choose to send the photos, you will still need to send the journal, unless you transcribe your journal reflections in a word document and email it to the project leader.

Step 4. After the project leader receives your photos and journal, she will contact you for a follow-up meeting to discuss your photos and journal reflections. 


\section{PhotoVoice Guidelines}

\section{Photo-Taking Tips}

\section{Tips for taking good photographs}

- $\quad$ Try different angles

- $\quad$ Try different points of view

- $\quad$ Keep the sun to your back, or to the side

- Is your subject in the center of the photo?

- Does your subject fill the photo?

\section{Tips for avoiding common mistakes}

- $\quad$ Keep your finger away from the lens

- Don't cover the flash

- Stand about three to eight feet away from your subject

- $\quad$ To prevent blurry pictures, hold your elbows close to your sides, and hold your breath when you press the shutter (button)

\section{PhotoVoice Ethics and Respect}

PhotoVoice is visual research. With visual research, you must keep certain guidelines in mind:

- Stay safe! Make sure you are "safe when you take the picture. For example:

- Stand on a solid surface

- Look before you step into or cross a street.

○ Be aware of things around you like traffic.

- Ask permission. Always ask permission before taking people's photos for this project. Ask them to sign a photo consent form (provided at the end of this packet). If people can be recognized in a photo, ask permission before showing their pictures.

- Be respectful. If certain people don't want their photo taken, respect their feelings.

- Be prepared. Be ready to explain about the project to family, friends, or strangers, if they ask what you are doing. A simple explanation is, "I am part of a PhotoVoice project exploring the health of our community. We are taking photographs of our lives and the community environment and talking about them with the project leaders. Thank you for letting me take your picture."

- When permission is not necessary. In a public place like a park, you can take someone's photo without permission, especially if they are far away and can't be recognized in the picture.

- Respect the lives and safety of others. When you take photos, think of people's safety first, and be respectful of their lives. For example:

$\circ$ If your friend is diabetic and the doctor told them not to eat sweets, avoid taking a picture of them eating cake.

- If your friend doesn't have a driver's license, avoid taking a picture of them driving a car down the street. 


\section{Using Your Camera}

- First, insert the batteries and the memory card into the camera.

- The power button is located on the top of the camera (on/off). Press it once to turn the camera on. Your camera is now ready to take pictures!

- To take a picture, press the large round button (the shutter) on the top of the camera.

- To zoom in, press the slider surrounding the shutter to the right.

- To zoom out, press the slider back to the left.

- To view pictures you have taken, press the button on the bottom right of the viewing screen (a rectangle with a "play" arrow inside of it).

- Use the right and left silver arrow keys to view all of your pictures.

- To delete images from the camera, find the image you wish to remove and press the button on the top right of the viewing screen (a trash can). Select "picture" and then press "OK." WARNING: Selecting "all" will delete all of the pictures you have taken so far.

- To return to picture-taking mode, press the button on the bottom right of the viewing screen again.

Your camera should adjust to the surroundings. At night or indoors, it will automatically flash. Outdoors (during the day) or in well-lit areas, the flash will not go off.

\section{Transferring Pictures to the Computer:}

- First, locate the cable that came with the camera. Attach the small end to the camera and the large end to an empty USB port in your computer.

- Turn the camera on after it is plugged into the computer. The computer will detect the camera and provide you with a list of options.

- Install the Kodak EasyShare software (only the first time) following the manufacturer's instructions provided with the camera.

- Follow the instructions given on screen to import images to the computer.

If you need assistance operating the camera, please email holly.huye@usm.edu or savanna.sims@eagles.usm.edu or call Holly Huye at 601-266-6023. 


\section{Photo Consent Form}

I am part of a PhotoVoice project exploring the health of my community environment. We are taking photographs of our community and people in the community that represent health or in need of a healthier lifestyle and talking about them with the project leader.

Please sign this form if you agree to let me take your photograph for this project and give your permission to copyright and use photographs that include your picture in presentations, as long as you are not identified by name or through other background information. If you would like a copy of this photo, please write down your address.

I agree to have my photo taken for this PhotoVoice project and hereby waive any right that I (and Minor) may have to inspect or approve the copy and/or finished product or products that may be used in connection therewith or the use to which it may be applied.

Name

Age (if under 18):

Address:

Signature

Consent of parent or legal guardian if above individual is a minor:

I consent and agree, individually and, as parent or legal guardian of the minor named above, to the foregoing terms and provisions. I hereby warrant that I am of full age and have every right to contract for the minor in the above regard. I state further that I have read the above information release and that I am fully familiar with the content.

Name of guardian

Relationship

Guardian signature

Name of photographer

Photographer signature

Date 


\section{APPENDIX C \\ DEMOGRAPHIC QUESTIONNAIRE \\ Demographic Questionnaire}

Please take a moment to answer the following questions placing a checkmark $(\checkmark)$ for the best choice. Remember, any information you share will remain anonymous and is only used to describe the participants as a group so no one is identified separately.

\section{Do you belong to a social or civic organization that participates in community} outreach?

Yes Please list name of organization(s) No

\section{What is your age range?}

$18-30$

$31-40$

$41-50$

51-60

$61-70$

$>71$

3. What is your occupation?

I am retired.

4. What is your marital status?

$\begin{array}{ll} & \text { Now Married } \\ & \text { Widowed } \\ & \text { Divorced } \\ & \text { Separated } \\ & \text { Never Married }\end{array}$

5. What was the last level of school you have completed?

Less than High School/GED

12th Grade (High School Grad/ GED)

Trade or VOC School 
__ Some College College Degree

Some Graduate or Professional School Graduate Level or Professional Degree

6. In general, would you say that your health is:

Excellent

Very good

Good

Fair

Poor

7. Of these income groups, which number best represents your household's total income in the last 12 months?

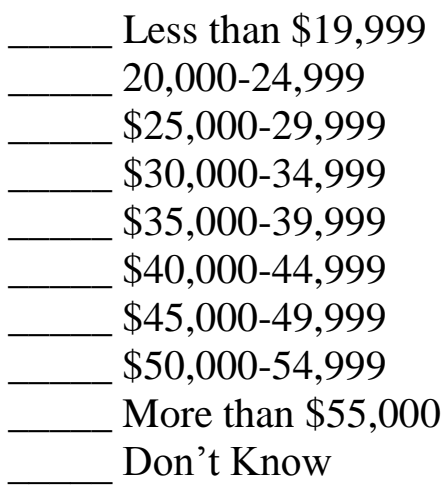

8. Do you consider yourself to be Hispanic or Latino?

A person of Mexican, Puerto Rican, Cuban, South or Central American, or other Spanish culture or origin, regardless of race.

Yes, Hispanic or Latino

Not Hispanic

9. What race do you consider yourself to be? Select one or more of the following. American Indian or Alaska Native

Asian

Black or African American

Native Hawaiian or Other Pacific Islander

White

More than two of the above

Don't Know 
APPENDIX D

INSTITUTIONAL REVIEW BOARD MATERIALS

\section{Women's Perceptions of the Health of the Delta Using Visual Depiction and Journal Reflections}

1. Project goals:

The goal of the research is to explore community health perceptions among women belonging to social and civic organizations using visual depiction and journal reflections in the Lower Mississippi Delta. In a previous study with women's social and civic organizations in the LMD, focus groups revealed a key theme: women's passion for the "Health of the Delta". In the present project, this theme will be explored further.

2. Protocol:

Participants will be recruited from a participant pool of prior research with women belonging to social and civic clubs in the LMD. A letter will be mailed to the women inviting them to participate (see Appendix 1). If participants cannot be obtained from that pool, participants will be recruited using an information recruitment flyer (see Appendix 2) at a Health Fair in the LMD community and may include word of mouth or snowball sampling methods. Qualitative research methods including key informant interviews and PhotoVoice methodology will be implemented.

Upon agreement to participate, the researcher will contact participants to set up an initial interview. During the interview the researcher will discuss the purpose of the research project and obtain informed consent (See Appendix 3). The researcher will then highlight the previous findings from the aforementioned research conducted and use a semi-structured interview guide (see Appendix 4) to facilitate discussion related to the Health of the Delta theme. At the end of the interview, the researcher will discuss the Photo Guiding Questions (see Appendix 5), instruct the participant on how to proceed with her responsibilities using PhotoVoice Guidelines adapted from "Doing Your Own PhotoVoice Project: A Guide" by Laura Lorenz, PhD and Barbara Webster (see Appendix 6), and collect demographic information (see Appendix 7), Participants will also receive a hard copy of the Guiding Questions and PhotoVoice Guidelines

Each participant will be provided a digital camera to take photographs of people, places, foods, and anything else she deems important that reflects healthy and unhealthy examples in her local community considering the framing questions. Participants who take any photos of people will be instructed to obtain a photo release form (see Appendix 8), which will be provided to the participants. In addition to the camera, a journal will also be provided. The purpose of the journal will allow participants to document specifics (i.e., date, time, place, etc.) and to write notes 
about their photos for the second interview. The participants will be allotted up to a 2week period to capture the state of the health environment. When the 2-week period ends and based on the participant's electronic capability, the participant will either 1) mail the camera or memory card along with the journal to the researcher using a prepaid envelope; or 2) upload the photos to an electronic source and email the photo files to the researcher as well as mail the journal to the researcher using a pre-paid envelope.

Once the researcher has received the journal and the photo files, the photos will be printed and participants will be contacted to set up an appointment to discuss their photos at a second interview. The researcher will use the photographs and journals to facilitate discussion about participants' perceptions of health in their communities during the interviews (see Appendix 9).

All interviews will be audio-recorded and transcribed. The transcripts will be analyzed for common themes relative the guiding framework and to the photographs taken.

Subject population: The study will consist of up to 10 participants living in the Lower Mississippi Delta (e.g., Greenwood, Greenville, Jonestown, MS areas). The population will consist of women over the age 18 .

Time: Interviews are expected to last one to one and a half hours. Photography and journaling is expected to take no more than 5 to 10 minutes per photo; Participants will be instructed to take up to 30 pictures.

Data gathering tools: Interview topic guides will be used to collect qualitative data. Each participant will receive a digital camera to take photographs as well as notebook/journal to log date, time, place and reflection of photo.

Benefits: Participants may gain valuable insights about the health of their community. Participants may also enjoy the creative components of this project, giving them the opportunity to express themselves both visually and in the written form. Additionally, the participants will be allowed to keep the camera (up to a $\$ 50$ value); a $\$ 50$ gift card to compensate for their participation time during this project will be given at the end of the second interview.

Risks: Risks associated with this research project may include participant's safety when taking pictures as well as the safety of their subjects. Participants will be instructed on safety and respect as described in the PhotoVoice Guidelines in Appendix 5. Other inconveniences to participants is the time it will take to take the picture, write in the journal, mailing the camera and journal to the researcher, and the attending the interviews. Only the researcher and research assistant will have access to the raw data collected. The data from the demographics survey will be entered into SPSS software and analyzed while interview recordings will be transcribed and coded 
for themes with corresponding photos. All data collection files will be destroyed at the end of the study.

3. Informed Consent:

The informed consent (see Appendix 3) will be given to participants during the initial interview for this project. This consent will state the purpose of the research and that the results may be used in presentations in appropriate venues. The informed consent will also state that the participant may discontinue the study at any time without penalty. 


\section{Enclosure Checklist}

(if not applicable, write N/A)

_ X__ Human Subjects Research Application (including complete narrative/protocol)

_ X__ RCR Certificate

N/A Letter from dissertation or thesis committee indicating approval of research proposal

_ X__ Survey/research instruments

N/A Permission letter from external agencies participating in the project

_ X__ Consent form to be used (long or short)

N/A Oral presentation

N/A Assent form for minors $<18$ years of age

N/A Cover letter or written instructions

N/A Letter to parents

N/A List or description of organizations to be contacted (school districts, hospitals, etc.) 


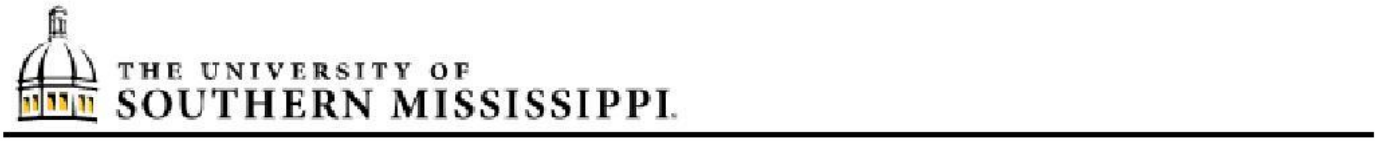

INSTITUTIONAL REVIEW BOARD

118 College Drive \#5147 I Hattiesburg, MS 39406-0001

Phone: 601.266.6820 | Fax: 601.266.4377| www.usm.edu/irb

\section{NOTICE OF COMMITTEE ACTION}

The project has been reviewed by The University of Southern Mississippi Institutional Review Board in accordance with Federal Drug Administration regulations (21 CFR 26, 111), Department of Health and Human Services (45 CFR Part 46), and university guidelines to ensure adherence to the following criteria:

- The risks to subjects are minimized.

- The risks to subjects are reasonable in relation to the anticipated benefits.

- The selection of subjects is equitable.

- Informed consent is adequate and appropriately documented.

- Where appropriate, the research plan makes adequate provisions for monitoring the data collected to ensure the safety of the subjects.

- Where appropriate, there are adequate provisions to protect the privacy of subjects and to maintain the confidentiality of all data.

- Appropriate additional safeguards have been included to protect vulnerable subjects.

- Any unanticipated, serious, or continuing problems encountered regarding risks to subjects must be reported immediately, but not later than 10 days following the event. This should be reported to the IRB Office via the "Adverse Effect Report Form".

- If approved, the maximum period of approval is limited to twelve months.

Projects that exceed this period must submit an application for renewal or continuation.

PROTOCOL NUMBER: 13052401

PROJECT TITLE: Women's Perceptions of the Health of the Delta Using Visual Depiction and Journal Reflections

PROJECT TYPE: New Project

RESEARCHER(S): Holly Huye, Ph.D.

COLLEGE/DIVISION: College of Health

DEPARTMENT: Nutrition and Food Systems

FUNDING AGENCY/SPONSOR: N/A

IRB COMMITTEE ACTION: Expedited Review Approval

PERIOD OF APPROVAL: $05 / 28 / 2013$ to $05 / 27 / 2014$

Lawrence A. Hosman, Ph.D.

Institutional Review Board 


\section{REFERENCES}

Airihihenbuwa, C. O., Kumanyika, S., Agurs, T. D., Lowe, A., Saunders, D. \& Morssink, C. B. (1996). Cultural aspects of African American eating patterns. Ethnicity \& Health,1(3), 245-271.

Alaimo, K., Packnett, E., Miles, R. A., \& Kruger, D. J. (2008). Fruit and vegetable intake among urban community gardeners. Journal of Nutrition Education and Behavior, 40(2), 94-101. doi:10.1016/j.jneb.2006.12.003

American Dietetic Association. (2011). Practice paper of the American Dietetic Association: Addressing racial and ethnic health disparities. Journal of the American Dietetic Association, 111(3), 446-456. doi:10.1016/j.jada.2011.01.024

Beydoun, M. A., \& Wang, Y. (2008). Do nutrition knowledge and beliefs modify the association of socioeconomic factors and diet quality among US adults? Preventative Medicine, 46, 145-153. doi:10.1016/j.ypmed.2007.06.016

Bhargava, A., \& Amialchuk, A. (2007). Added sugars displaced the use of vital nutrients in the National Food Stamp Program Survey. Journal of Nutrition, 137, 453-460.

Carver, A., Timperio, A., \& Crawford, D. (2008). Playing it safe: The influence of neighborhood safety on children's physical activity_A review. Health \& Place, 14, 217-227. doi:10.1016.j.healthplace.2007.06.004

Castellanos, D. C., Connell, C., \& Lee, J. (2011). Factors affecting weight gain and dietary intake in Latino males residing in Mississippi: A preliminary study. Hispanic Health Care International, 9(2), 91-98. doi:10.1891/1540-4153.9.2.91

Castellanos, D. C., Downey, L., Graham-Kresge, S., Yadrick, K., Zoellner, J., \& Connell, C. L. (2013). Examining the diet of post-migrant Hispanic males using the 
precede-proceed model: Predisposing, reinforcing, and enabling dietary factors. Journal of Nutrition Education and Behavior, 45, 109-118.

doi:10.1016/j.jneb.2012.05.013

Catalani, C., \& Minkler, M. (2010). Photovoice: A review of the literature in health and public health. Health Education and Behavior, 37, 424-451.

doi:10.1177/1090198109432084

Centers for Disease Control and Prevention. (2012). Mississippi's Response to Obesity: State Nutrition, Physical Activity, and Obesity Profile. Retrieved from http://www.cdc.gov/obesity/stateprograms/fundedstates/mississippi.html

Centers for Disease Control and Prevention. (2013). Adult Obesity Facts. Retrieved from http://www.cdc.gov/obesity/data/adult.html

Champagne, C. M., Casey, P. H., Connell, C. L., Stuff, J. E., Gossett, J. M., Harsha, D. W., .. \& Bogle, M. L. (2007). Poverty and food intake in rural America: Diet quality is lower in food insecure adults in the Mississippi Delta. Journal of the American Dietetic Association, 107, 1886-1894. doi:10.1016/j.jada.2007.08.003

Charmaz, K. (2000). Grounded theory: Objectivist and constructivist methods. In N. K. Denzin \& Y. Lincoln (Eds.), Handbook of qualitative research (pp. 509-535). Thousand Oaks, CA: Sage.

Chilton, M., Rabinowich, J., Council, C., \& Breaux, J., (2009). Witnesses to hunger: Participation through PhotoVoice to ensure the right to food. Health and Human Rights, 11(1), 73-85.

Coleman-Jensen, A., Nord, M., Andrews, M., \& Carlson, S. (2011). Household Food Security in the United States in 2010. Retrieved from 
http://www.ers.usda.gov/publications/err-economic-researchreport/err125.aspx\#.Uqi4VCdDpk0

Connell, C. L., Yadrick, M. K., Simpson, P., Gossett, J., McGee, B. B., \& Bogle, M. L. (2007). Food supply adequacy in the Lower Mississippi Delta. Journal of Nutrition Education and Behavior, 39, 77-83. doi:10.1016/j.jneb.2006.10.007

Corbin, J., \& Strauss, A. (1990). Grounded theory research: Procedures, canons, and evaluative criteria. Zeitschrift fur Soziologie, 19(6), 418-427.

Creswell, J.W. (2005). Educational research: Planning, conducting, and evaluating quantitative and qualitative research. $\left(2^{\text {nd }}\right.$ ed.) Upper Saddle River, NJ: Pearson.

Curry, K. R. (2000). Multicultural competence in dietetics and nutrition. Journal of the American Dietetic Association, 100(10), 1142-1143.

Dietz, W. (1995). Does hunger cause obesity? Pediatrics, 95(5), 766-767.

Ellison, C. G. (1995). Race, religious involvement and depressive symptomatology in a southeastern U. S. community. Social Science and Medicine, 40(11), 1561-1572.

Everson, S. A., Maty, S, C., Lynch, J. W., \& Kaplan, G. A. (2002). Epidemiologic evidence for the relation between socioeconomic status and depression, obesity, and diabetes. Journal of Psychosomatic Research, 53, 891-895.

Farley, T. A., Rice, J., Bodor, J. N., Cohen, D. A., Bluthenthal, R. N., \& Rose, D. (2009). Measuring the food environment: Shelf space of fruits, vegetables, and snack foods in stores. Journal of Urban Health: Bulletin of the New York Academy of Medicine, 86(5), 672-682. doi:10.1007/s11524-009-9390-3

Findholt, N. E., Michael, Y.L., Davis, M. M., \& Brogoitti, V. W. (2010). Environmental influences on children's physical activity and diets in rural Oregon: Results of a 
youth photovoice project. Online Journal of Rural Nursing and Health Care, 10(2), 11-20.

Fontenot, K., Singelmann, J., Slack, T., Siordia, C., Poston, D. L., \& Saenz, R. (2010). Understanding falling poverty in the poorest places: An examination of the experience of the Texas Borderland and Lower Mississippi Delta, 1990-2000. Journal of Poverty, 14, 216-236. doi:10.1080/10875541003712183

Galanti, G. (2003). The Hispanic family and male-female relationships: An overview. Journal of Transcultural Nursing, 14, 180-185. doi:10.1177/1043659603014003004

Gray, P. A. (1991). Economic development and African Americans in the Mississippi Delta. Rural Sociology, 56, 238-246.

Haldeman, L. A., Gruber, K. J., \& Ingram, K. P. (2007). Determinants of food security and diet among rural and urban Latino/Hispanic immigrants. Journal of Hunger and Environmental Nutrition, 2(4), 67-83. doi:10.1080/19320240802032503

Hannay, J., Dudley, R., Milan, S., \& Leibovitz, P. K. (2013). Combining photovoice and focus groups: Engaging Latina teens in community assessment. American Journal of Preventative Medicine, 44, S215-S224. doi:10.1016/j.amepre.2012.11.011

Haskell, W. L., Lee, I., Pate, R. R., Powell, K. E. \& Blair, S. N. (2007). Physical activity and public health: Updated recommendation for adults from the American College of Sports Medicine and the American Heart Association. Circulation, 116(9), 1081-1093. doi:10.1249.mss.0b013e3180616b27

Huye, H. F., Connell, C. L., Crook, L. B., Yadrick, K., \& Zoellner, J. (2014). Using the RE-AIM framework in formative evaluation and program planning for a 
nutrition intervention in the Lower Mississippi Delta. Journal of Nutrition Education and Behavior, 46, 34-42. doi:10.1016/j.jneb.2013.09.006

Jones, R., Thornell, J., \& Hamon, G. (1992). Educational attainment in the Delta. In A. G. Cosby, M. W. Brackin, T. D. Mason, \& E. R. McCulloch (Eds.), A social and economic portrait of the Mississippi Delta (90-108). Mississippi State, MS: Mississippi State University.

Kaiser Family Foundation. (2011). State Health Facts: Overweight and Obesity Rates for Adults by Race/Ethnicity. Retrieved from http://kff.org/other/state-indicator/adultoverweightobesity-rate-by-re/

Kittler, P. G., \& Sucher, K. P. (1998). Food and culture (5 ${ }^{\text {th }}$ ed.). Florence, KY: Cengage Learning.

Kreuter, M. W., Lukwago, S. N., Bucholtz, D. C., Clark, E. M., \& Sanders-Thompson, V. (2002). Achieving cultural appropriateness in health promotion programs:

Targeted and tailored approaches. Health Education and Behavior, 30(2), 133146. doi: $10.1177 / 1090198102551021$

Krueger, R. A. (2013). Focus group interviewing. Minneapolis, MN: Richard A. Krueger. Kumpfer, K. L., Alvarado, R., Smith, P., \& Bellamy, N. (2002). Cultural sensitivity and adaptation in family-based prevention interventions. Prevention Science, 3(3), 241-246.

Landrine, H., \& Klonoff, E. A. (1992). Culture and health-related schemas: A review and proposal for interdisciplinary integration. Health Psychology, 11, 267-276.

Lara, M., Gamboa, C., Kahramanian, M. I., Morales, L. S., \& Bautista, D. E. H. (2005). Acculturation and Latino health in the United States: A review of the literature 
and its sociopolitical context. Annual Reviews of Public Health, 26, 367-397. doi:10.1146/annurev.publhealth.26.021304.144615

Larson, N. I., Story, M. T., \& Nelson, M. C. (2009). Neighborhood environments: Disparities in access to healthy foods in the U.S. American Journal of Preventative Medicine, 36(1), 74-81. doi:10.1016/j.amepre.2008.09.025

Lentz, R. D. \& Oden, M. D. (2001). Digital divide or digital opportunity in the Mississippi Delta region of the US. Telecommunications Policy, 25(5), 291-313.

Lindsay, A. C., Sussner, K. M., Kim, J., \& Gortmaker, S. (2006). The role of parents in preventing childhood obesity. The Future of Children, 16(1), 169-186.

Lopez, E. D. S., Eng, E., Randall-David, E., \& Robinson, N. (2005). Quality-of-life concerns of African American breast cancer survivors within rural North Carolina: Blending the techniques of PhotoVoice and grounded theory. Qualitative Health Research, 15, 99-115. doi:10.1177/1049732304270766

Lower Mississippi Delta Nutrition Intervention Research Consortium. (2010). High prevalence of food insecurity and hunger in households in the rural Lower Mississippi Delta. The Journal of Rural Health, 20(2), 173-180.

Lumpkins, C. Y., Greiner, K. A., Daley, C., Mabachi, N. M., \& Neuhaus, K. (2013). Promoting healthy behavior from the pulpit: Clergy share their perspectives on effective health communication in the African American church. Journal of Religious Health, 52, 1093-1107. doi:10.1007/s10943-011-9533-1

Madill, A., Jordan, A., \& Shirley, C. (2000). Objectivity and reliability in qualitative analysis: Realist, contextualist, and radical constructionist epistemologies. British Journal of Psychology, 91(1), 1-20. 
Marin, G. (1993). Defining culturally appropriate community interventions: Hispanics as a case study. Journal of Community Psychology, 21, 149-161.

Martin, K. S., \& Ferris, A. M. (2007). Food insecurity and gender are risk factors for obesity. Journal of Nutrition Education and Behavior, 39(1), 31-36. doi: 10.1016/j.jneb.2006.08.021

Martin, N., Garcia, A. C., \& Leipert, B. (2010). PhotoVoice and its potential use in nutrition and dietetic research. Canadian Journal of Dietetic Practice and Research, 71, 93-97. doi: 10.3148/71.2.2010.93

Maton, K. I. \& Wells, E. A. (1995). Religion as a community resource for well-being: Prevention, healing, and empowerment pathways. Journal of Social Issues, 51(2), 177-193.

McArthur, L. H., Anguiano, R. P. V., \& Nocetti, D. (2001). Maintenance and change in the diet of Hispanic immigrants in eastern North Carolina. Family and Consumer Sciences Research Journal, 29(4), 309-335.

McCabe-Sellers, B. J., Bowman, S., Stuff, J. E., Champagne, C. M., Simpson, P. M., \& Bogle, M. L. (2007). Assessment of the diet quality of US adults in the Lower Mississippi Delta. American Journal of Clinical Nutrition, 86, 697-706.

Mirvis, D. M., Steinberg, S., \& Brown, L. (2004). Health improvement in the Lower Mississippi River Delta: Opportunities and challenges [Powerpoint slides]. Retrieved from http://www.msdh.state.ms.us/msdhsite/_static/resources/3114.pdf Mississippi State Department of Health. (2012a). Mississippi Delta Region: Burden of Chronic Diseases. Retrieved from http://msdh.ms.gov/msdhsite/ _static/resources/5048.pdf 
Mississippi State Department of Health. (2012b). 2012 Behavioral Risk Factor Surveillance Survey: Mississippi Annual Prevalence Report. Retrieved from http://www.msdh.state.ms.us/brfss/brfss2012ar.pdf

National Institutes of Health. (2002). Strategic Research Plan and Budget to Reduce and Ultimately Eliminate Health Disparities. Retrieved from http://www.nimhd.nih.gov/our_programs/strategic/pubs/VolumeI_031003EDrev. pdf

Neill, C., Leipert, B. D., Garcia, A. C., \& Kloseck, M. (2011). Using photovoice methodology to investigate facilitators and barriers to food acquisition and preparation by rural older women. Journal of Nutrition in Gerontology and Geriatrics, 30(3), 225-247. doi: 10.1080/21551197.2011.591268

Nelsen, H. M., Yokley. R. L., \& Nelsen, A. K. (Eds). (1971). The Black Church in America. New York, NY: Basic Books.

Nykiforuk, C., Vallianatos, H., \& Nieuwendyk, L. M. (2011). Photovoice as a method for revealing community perceptions of the built and social environment. International Journal of Qualitative Methods, 10(2), 103-124.

Parham, G. P., \& Scarinci, I. C. (2007). Strategies for achieving healthy energy balance among Blacks in the Mississippi Delta. Preventing Chronic Disease, 4(4), 1-10.

Perez-Escamilla, R. (2009). Dietary quality among Latinos: Is acculturation making us sick? Journal of the American Dietetic Association, 109(6), 988-991. doi: 10.1016/j.jada.2009.03.014

Perry, C. L., Luepker, R. V., Murray, D. M., Kurth, C., Mullis, R., Crockett, S., \& Jacobs, D. R. (1988). Parent involvement with children's health promotion: The 
Minnesota Home Team. American Journal of Public Health, 78, 1156-1160.

Pollard, T. M., Steptoe, A., \& Wardle, J. (1998). Motives underlying healthy eating: Using the Food Choice Questionnaire to explain variation in dietary intake. Journal of Biosocial Science, 30, 165-179.

Quandt, S. A., Shoaf, J. I., Tapia, J., Hernandez-Pelletier, M., Clark, H. M., \& Arcury, T. A. (2006). Experiences of Latino immigrant families in North Carolina help explain elevated levels of food insecurity and hunger. Journal of Nutrition, 136, 2638-2644.

Rodriguez, N. (2012). New southern neighbors: Latino immigration and prospects for intergroup relations between African-Americans and Latinos in the South. Latino Studies, 10(1-2), 18-40.

Ross, J. C., \& Wheeler, R. (1971). Black Belonging. Westport, CT: Greenwood Press. Smith, J., Lensing, S., Horton, J. A., Lovejoy, J., Zaghloul, S., Forrester, I., McGee, B. B., \& Bogle, M. L. (1999). Prevalence of self-reported nutrition-related health problems in the Lower Mississippi Delta. American Journal of Public Health, 89, $1418-1421$.

Strack, R. W., Lovelace, K. A., Jordan, T. D., \& Holmes, A. P. (2010). Framing photovoice using a social-ecological logic model as a guide. Health Promotion Practice, 11, 629-636. doi:10.1177/1524839909355519

Stuff, J. E., Casey, P. H., Szeto, K. L., Gossett, J. M., Robbins, J. M., Simpson, P. M., ... \& Bogle, M. L. (2004). Household food insecurity is associated with adult health status. Journal of Nutrition, 134, 2330-2335.

Taylor, R. J., Chatters, L. M., Hardison, C. B., \& Riley, A. (2001). Informal social 
support networks and subjective well-being among African Americans. Journal of Black Psychology, 27(4), 439-463.

Thomas, D. (2008). The digital divide: What schools in low socioeconomic areas must teach. The Delta Kappa Gamma Bulletin, Summer 2008, 12-17.

Thomas, H. C., \& Irwin, J. D. (2013). Using PhotoVoice with at-risk youth in a community-based cooking program. Canadian Journal of Dietetic Practice and Research, 74, 14-20. doi:10.3148/74.1.2013.14

Torres, M. E., Meetze, E. G., Smithwick-Leone, J. (2013). Latina voices in childhood obesity: A pilot study using photovoice in South Carolina. American Journal of Preventative Medicine, 44, S225-S231. doi:10.1016/j.amepre.2012.11.020

Triandis, H. C., Marin, G., Lisansky, J., \& Betancourt, H. (1984). Simpatia as a cultural script of Hispanics. Journal of Personality and Social Psychology, 47, 1363-1375.

U.S. Census Bureau. (2013a). State and County Quick Facts: Mississippi. Retrieved from http://quickfacts.census.gov/qfd/states/28000.html

U.S. Census Bureau. (2013b). State and County Quick Facts: USA. Retrieved from http://quickfacts.census.gov/qfd/states/00000.html

University of Memphis Regional Economic Development Center. (2002). Demographic Profile by Development District and by County for Lower Mississippi Delta Region Tables and Maps Census 1990-2000. Retrieved from http://planning.memphis.edu/redc-publications_files/FINALMississippi\%20Delta.pdf

Valera, P., Gallin, J., Schuk, D., \& Davis, N. (2009). “Trying to eat healthy:” A photovoice study about women's access to healthy food in New York City. 
Affilia, 24, 300-314. doi: 10.1177/0886109909337378

Wang, Y., \& Beydoun, M. A. (2007). The obesity epidemic in the United StatesGender, age, socioeconomic, racial/ethnic, and geographic characteristics: A systematic review and meta-regression analysis. Epidemiologic Reviews, 29, 6-28. doi:10.1093/epirev/mxm007

Wang, C., \& Burris, M. A. (1997). Photovoice: Concept, methodology, and use for participatory needs assessment. Health Education and Behavior, 24(3), 369-387.

Wang, C. C., \& Pies, C. A. (2004). Family, maternal, and child health through photovoice. Maternal and Child Health Journal, 8(2), 95-102.

Wang, C. C. (1999). Photovoice: A participatory action research strategy applied to women's health. Journal of Women's Health, 8(2), 185-192.

Wang, C. C., Yi, W. K., Tao, Z. W., \& Carovano, K. (1998). Photovoice as a participatory health promotion strategy. Health Promotion International, 13(1), 75-86.

Warburton, D. E. R., Nicol, C. W., \& Bredin, S. S. D. (2006). Health benefits of physical activity: The evidence. Canadian Medical Association Journal, 174(6), 801-809. doi:10.1503/cmaj.051351

Williams, R., Hewison, A., Stewart, M., Liles, C., \& Wildman, S. (2012). 'We are doing our best:' African and African-Caribbean fatherhood, health and preventative primary care services, in England. Health and Social Care in the Community, 20(2), 216-223. doi:10.1111/j.1365-2524.2011.01037.x

Woolford, S. J., Khan, S., Barr, K.L.C., Clark, S. J., Strecher, V. J., \& Resnicow, K. (2012). A picture may be worth a thousand texts: Obese adolescents' perspectives 
on a modified photovoice activity to aid weight loss. Childhood Obesity, 8(3), 230-236. doi: 10.1089/chi.2011.0095

Yeh, M., Ickes, S. B., Lowenstein, L. M., Shuval, K., Ammerman, A. S., Farris, R., \& Katz, D. L. (2008). Understanding barriers and facilitators of fruit and vegetable consumption among a diverse multi-ethnic population in the USA. Health Promotion International, 23(1), 42-51. doi:10.1093/heapro/dam044

Zoellner, J., Connell, C., Bounds, W., Crook, L., \& Yadrick, K. (2009). Nutrition literacy status and preferred nutrition communication channels among adults in the Lower Mississippi Delta. Preventing Chronic Disease, 6(4), 1-11.

Zoellner, J., Hill, J. L., Zynda, K., Sample, A. D., \& Yadrick, K. (2012). Environmental perceptions and objective walking trail audits inform a community-based participatory research walking intervention. International Journal of Behavioral Nutrition and Physical Activity, 9(6), 1-11. doi:10.1186/1479-5868-9-6 